\title{
MODULAR SCHUR FUNCTIONS
}

\author{
GRANT WALKER
}

\begin{abstract}
A new family of symmetric functions is considered. These functions are analogous to the classical Schur functions, but depend on an integer modulus $p \geq 2$, as well as on a partition $\lambda$. In the case where $p$ is prime, certain of these functions are shown to be irreducible characters of the general linear group $G L(n, K)$ in the natural characteristic $p$ of the field $K$. This dualises a wellknown criterion of G. D. James for such characters to be given by classical Schur functions.
\end{abstract}

The problem of determining the simple $p$-modular polynomial characters of the general linear group $G L(n, K)$ over an algebraically closed field $K$ of prime characteristic $p$ is (justly) regarded as both important and very difficult. In one sense this is surprising, because it is well known how to construct a complete set of simple modules explicitly, either as quotients of Weyl modules $V(\lambda)$, or as submodules of their duals, the corresponding Schur modules $H^{0}(\lambda)$. (A recent treatment is given in $[14, \S 3]$.) The difficulty lies in extracting formulae for the characters, or even in calculating the dimensions of the modules, from these general constructions. The same situation arises with regard to the simple modular representations of the symmetric group; and indeed, G. D. James has shown that the two problems are, in effect, equivalent.

Some special cases of this problem are, however, tractable. The (formal) character of the Weyl and Schur modules with highest weight $\lambda$ is the Schur function $s_{\lambda}$, and for a field $K$ of characteristic 0 these are the simple characters. James and Murphy $[6,9]$ have given a necessary and sufficient condition (first conjectured by R. W. Carter) for $s_{\lambda}$ to be a simple modular character of $G L(n, K)$ for all sufficiently large $n$. The purpose of this article is to show that, for odd primes $p$, there is a natural general formula for a second family of simple modular characters, also indexed by partitions satisfying the Carter criterion, and to determine the highest weight of these simple characters. The main result is Theorem 4.4. The results are valid also for $p=2$, but give no new information in this case.

These simple characters belong to a family of symmetric functions which we call modular Schur functions. As explained in [4, §3], we consider an endomorphism $\omega^{\prime}$ of the ring of symmetric functions $\Lambda$. This is defined on the generator $h_{d}$, the complete symmetric function of degree $d$, by truncation at the $p$ th powers of the variables. Here $p$ can be any integer $\geq 2$, not necessarily prime. The image $\omega^{\prime}(f)=f^{\prime}$ of $f$ is, by definition, the 'modular' symmetric

Received by the editors November 21, 1993.

1991 Mathematics Subject Classification. Primary 05E05, 20C20; Secondary 05E10, 20C30. 
function corresponding to $f$. In the case $p=2, \omega^{\prime}=\omega$ is the well-known involution $[13, \S 1]$ which exchanges the complete symmetric functions with the elementary symmetric functions. An important fact about the modular Schur functions is that, when $p$ is prime, the column regular part of the decomposition matrix for modular Schur functions in terms of simple characters is the same as for 'ordinary' Schur functions. This is an essential step in the argument (Proposition 4.2). However, the modular Schur functions are usually only virtual characters, as negative integers appear in the column singular part of the decomposition matrix.

We now summarise the contents of this paper.

The short preliminary Section 0 consists of background material on the combinatorics of partitions that is needed throughout the paper, and establishes notation and conventions. Nearly all of this is standard, but the definition of horizontal partition is new, and is essential for the proof of the main theorem.

Much of Section 1 is based on an observation of I. G. Macdonald [4, Proposition 3.15], which is restated here as Proposition 1.2. It follows from this that, if we consider symmetric functions with rational coefficients, $\omega^{\prime}$ becomes a ring automorphism. By considering the modular Schur functions which are indexed by all partitions of a given integer $d$, and expressing them in terms of ordinary Schur functions, we can thus define a 'transition matrix' $M\left(s^{\prime}, s\right)$ with integer entries and nonzero determinant. This matrix is symmetric, and Macdonald's observation gives us its eigenvalues, which are powers of $1-p$, and its eigenvectors, which form the character table of the symmetric group $S_{d}$. In the case $p=2$, the matrices $M\left(s^{\prime}, s\right)$ simplify to 'conjugation' matrices, since $s_{\lambda}^{\prime}=s_{\lambda^{\prime}}$. We also reformulate the main conjecture of [4] in this context, and prove it in degrees $d<2 p$.

In Section 2, we show that the transition matrix $M\left(s^{\prime}, s\right)$ exhibits a 'block theory' of the type which occurs in the representation theory of the symmetric group. That is, the modular Schur function $s_{\lambda}^{\prime}$ is an integer linear combination of Schur functions $s_{\mu}$, where $\mu$ runs over the set of partitions of $d$ with the same core as $\lambda$. Thus, one of the most important features of the modular representation theory of the general linear and symmetric groups occurs here at a purely combinatorial level. The main tool used in the proof is an operation $\psi_{p}$ on symmetric functions, which we call the $p$-quotient map: $\psi_{p}$ is adjoint to the $p$ th power (or Frobenius) map $\psi^{p}$.

Inside a block, we show that the $(\lambda, \mu)$ th entry $\left\langle s_{\lambda}^{\prime}, s_{\mu}\right\rangle$ of $M\left(s^{\prime}, s\right)$ depends only on the signs and quotients of $\lambda$ and $\mu$. The quotient is an ordered $p$ tuple of partitions, the sum of the parts of all $p$ partitions being the weight $w$ of the block. Given $p$ and $w$, we may consider the transition matrices from Schur functions to modular Schur functions for all blocks of given weight $w$ by choosing a fixed order for the quotients. Proposition 2.9 shows that all these matrices are identical up to the signs of the entries. In some cases, we can do better: J. C. Scopes [17] has given a sufficient condition for two blocks of symmetric groups $S_{d}$ and $S_{d^{\prime}}$ to be Morita equivalent, and we show in Proposition 2.12 that, when this combinatorial condition is satisfied, the matrices $M\left(s^{\prime}, s\right)$ for the two blocks are actually identical when the partitions in each block are ordered lexicographically.

We conclude Section 2 by showing that the functions $s_{\lambda}$ and $s_{\lambda}^{\prime}$ behave in a similar way under contragredient duality. This also simplifies the task of 
checking Conjecture 1.4 in particular cases, and we illustrate this by proving the conjecture for $n=3$ variables.

In Section 3, we study two classes of partitions $\lambda$ for which the highest weight of the modular Schur function $s_{\lambda}^{\prime}$ is column regular (i.e. $p$-restricted) and can be explicitly calculated. One such class of partitions, which we call row stable, consists of the partitions whose (nonzero) parts differ by at least $p-1$. The other class, which we call horizontal, may be defined in various ways. One definition is that only 'horizontal' (rim) $p$-hooks can be removed from the diagram; another is that, in each column of the diagram, either all or none of the hook lengths are divisible by $p$. For example, partitions satisfying Carter's criterion are horizontal, and the 1-part partitions $(d)$ and the 'Steinberg' partitions $((n-1)(p-1),(n-2)(p-1), \ldots, 2(p-1), p-1)$ are examples of both types. The function $s_{(d)}^{\prime}=h_{d}^{\prime}$, the $p$-truncated complete symmetric function of degree $d$. It is well known (see e.g. [1, Theorem 6.1]) that the truncated symmetric power $T^{d}(E)$ of the natural module $E$ for $G L(n, K)$ is simple for all $d$, with formal character $h_{d}^{\prime}$, and it was an attempt to generalise this result that led us to consider modular symmetric functions in the first place.

The main technical objective of Section 3 is achieved in Proposition 3.8. Essentially this proves that certain entries in the transition matrices $M\left(s^{\prime}, s\right)$ are 0 . The trick is to establish the result directly for the case where the core of $\lambda$ is a Steinberg partition, and then to extend to other blocks using the work of Section 2.

All the results of Sections 1, 2, and 3 are valid for an arbitrary modulus $p \geq 2$. In Section 4 we restrict $p$ to be prime, so as to obtain applications to modular representation theory. A key step is Proposition 4.2, which shows that the modular and ordinary Schur functions have the same column regular decomposition numbers. For the proof we use Brauer characters: the reader familiar with the Schur functor may prefer a different argument. This fact is then combined with the Proposition 3.8 to read off, from the work of James on Specht modules, the cases where a horizontal partition $\lambda$ leads to a simple character $s_{\lambda}^{\prime}$ of $G L(n, K)$, so proving Theorem 4.4.

In the course of this work, a number of questions have arisen that we have been unable to answer. First, are there any cases, other than those given by Theorem 4.4, where $s_{\lambda}^{\prime}$ is a simple character of $G L(n, K)$ for all sufficiently large $n$ ? Calculations for blocks of low weight suggest very strongly that there are not, as this requires the coincidence of two rare occurrences: the dual Specht module $S_{\lambda}$ must be simple, and $s_{\lambda}^{\prime}$ must have no column singular decomposition numbers. However, the simple Specht modules have not been fully determined, and although a sufficiently general test for $s_{\lambda}^{\prime}$ to have at least one column singular decomposition number would settle the matter, we have not succeeded in finding a suitable condition.

A second question concerns labelling. The relation between the row regular partition $\lambda$ and the column regular partition $\mu$ in our main theorem, Theorem 4.4 , is that $\mu=l(\lambda)^{\prime}$, where $l$ is the involution on the set of all row regular partitions that corresponds to multiplying the corresponding simple character of the symmetric group by the sign character. The (notorious) conjecture of Mullineux [15] gives a combinatorial algorithm for $l$, and it is traditional to confirm that results in this area are consistent with the conjecture of Mullineux. 
Although we strongly believe that this is true for Theorem 4.4, we have not been able to prove it.

This work was originated in collaboration with Stephen R. Doty, and the first person plural is retained throughout in recognition of his contribution. I am grateful for support from Loyola University of Chicago for a visit in 1992, during which some of the ideas here were worked out.

It is a pleasure to thank my colleagues at Manchester for helpful suggestions at critical moments in this work, and also to thank Stuart Martin for valuable discussions, for sharing his unpublished computations, and for writing [14]. The author's debt to the work of Ian Macdonald and Gordon James will be obvious, and their books [13], [8] are cited as standard references throughout.

\section{Partitions}

The representations and symmetric functions with which we are concerned are naturally indexed by partitions. For the reader's convenience, we collect here the various definitions that we need relating to partitions. General references are [13] and [8].

A partition $\lambda$ of an integer $d \geq 0$ is a monotonic decreasing sequence $\lambda_{1}, \lambda_{2}, \ldots$ of integers $\geq 0$ whose sum is $d$. The nonzero terms of the sequence are called the parts, the sum $d=|\lambda|$ the degree, and the number $l=l(\lambda)$ of nonzero parts the length of the partition. The sum $\lambda+\mu$ of two partitions and the product $k \lambda$ of a partition by an integer $k \geq 0$ have the usual meanings for sequences. We usually omit zero parts, and write repeated parts as powers: thus the partitions of 3 are written as $(3),(2,1)$ and $\left(1^{3}\right)$. We write $\leq$ for the (total) lexicographic order, and $\unlhd$ for the (partial) dominance order [8, p. 23].

We use the same symbol $\lambda$ for a partition and for its (Young) diagram, for which we use matrix conventions. The transpose of the diagram is the diagram of the conjugate partition $\lambda^{\prime}$. The elements of the diagram are called its nodes. A tableau is obtained by replacing the nodes by positive integers, with no restriction. The tableau is semistandard if these numbers increase monotonically along rows and strictly down columns. The content of a tableau is the sequence whose $k$ th term is the number of times $k$ appears.

The hook length $h(i, j)$ of the $(i, j)$ node is the number of nodes $\left(i^{\prime}, j^{\prime}\right)$ with $i^{\prime}=i, j^{\prime} \geq j$ or $j^{\prime}=j, i^{\prime} \geq i[8, \mathrm{p}$. 55]. The rim of the diagram is the set of nodes $(i, j)$ such that $(i+1, j+1)$ is not a node. The $(i, j)(\mathrm{rim})$ hook has length $h(i, j)$ and consists of the rim nodes $\left(i^{\prime}, j^{\prime}\right)$ with $i^{\prime} \geq i$ and $j^{\prime} \geq j$. Its height $h$ is the maximum value of $i^{\prime}-i$ for these nodes (i.e. one less than the 'leg length of the $(i, j)$-hook').

Let $p \geq 2$ be an integer, which need not be prime. An integer $d \geq 0$ can be written uniquely as $p q+r$, where $0 \leq r \leq p-1$. This can be generalised to partitions, as follows.

A $p$-hook is a rim hook of length $p$. By successive removal of a sequence of $p$-hooks from the diagram $\lambda$, a unique diagram $\nu$ is reached, from which no further $p$-hook can be removed. This is the core of $\lambda$, which generalises the residue $r$. A partition is a core if and only if no hook length is divisible by $p$ $[8,2.7 .40]$.

The $p$-hooks which have been removed determine the quotient of $\lambda$, which is an ordered $p$-tuple of partitions $(\lambda(0), \lambda(1), \ldots, \lambda(p-1))$. The core and 
quotient determine the partition uniquely. Conversely, for any partition which is a core, every $p$-tuple of partitions is the quotient of a partition with that core. The sum of the degrees of the components $\lambda(i)$ of the quotient is the weight $w=w(\lambda)$ of $\lambda$ : this is the number of $p$-hooks that are removed from $\lambda$ to leave the core $\nu$, so that $p w=d-d^{\prime}$, where $d^{\prime}$ is the degree of $\nu$. The set of all partitions with the same degree and the same core is called a block of weight $w$.

All of this can be conveniently expressed using 'abacus' notation [8, 2.7]. For any $b \geq l$, the length of $\lambda$, the strictly decreasing sequence

$$
\left(\lambda_{1}+b-1, \ldots, \lambda_{i}+b-i, \ldots, \lambda_{b}\right)
$$

of integers $\geq 0$ is called a sequence of $\beta$-numbers for $\lambda$ (cf. [12]). If $b=l$, the $\beta$-numbers are the first column hook lengths of $\lambda$, but for any $b$ this set of numbers determines $\lambda$ uniquely. The 'abacus' has $p$ 'runners', numbered $0,1, \ldots, p-1$, on which 'bead positions' are labelled as below.

$$
\begin{array}{cccc}
0 & 1 & \ldots & p-1 \\
p & p+1 & \ldots & 2 p-1 \\
2 p & 2 p+1 & \ldots & 3 p-1 \\
\vdots & \vdots & \vdots & \vdots
\end{array}
$$

A partition $\lambda$ is shown on the abacus by a set of $b$ 'beads' which occupy the positions labelled by the set of $\beta$-numbers. In this notation, removal of a $p$ hook corresponds to moving a bead one place up on its runner. Thus the core is shown by moving all the beads up as far as possible, while the quotient records the number of places each bead moves in this process. (It is to be understood that, as on a real abacus, two beads cannot occupy the same position, and the order of the beads on a runner is fixed.) More precisely, the moved-up bead positions are a set of $\beta$-numbers for the core, while $\beta$-numbers for the quotient $\lambda(0), \lambda(1), \ldots, \lambda(p-1)$ are obtained from the original bead positions (the $\beta$ numbers for $\lambda$ ) as follows. Number the rows of the abacus from the top as $0,1,2, \ldots$, and let $0 \leq k \leq p-1$. Then the numbers of the rows containing beads on the $k$ th runner are a set of $\beta$-numbers for $\lambda(k)$.

The quotient is fixed by the choice of the number of beads $b \bmod p$. If we change the value of $b$, then the components of the quotient are permuted cyclically. No particular convention for choosing $b$ seems ideal for all purposes, so we shall choose $b$ to suit the occasion.

The abacus is also used to associate a sign $\varepsilon(\lambda)= \pm 1$ to the partition $\lambda$. This is the sign of the permutation of $b$ objects obtained by comparing the order of the beads when placed as a set of $\beta$-numbers for $\lambda$ with their order when moved up the runners to give a set of $\beta$-numbers for its core $\nu$. The sign $\varepsilon(\lambda)=(-1)^{h}$, where $h$ can be taken as the sum of the heights of any sequence of $p$-hooks that are removed from $\lambda$ to leave $\nu$. Since the sum of the heights of any $p$-hook and its conjugate is $p-1$, the sign of the conjugate of a partition of weight $w$ is given by $\varepsilon\left(\lambda^{\prime}\right)=(-1)^{(p-1) w} \varepsilon(\lambda)$.

For applications to representation theory, we need to distinguish certain special types of partition. A partition is row regular if its diagram does not have $p$ equal rows, and column regular (or p-restricted) if it does not have $p$ equal columns. Row singular means not row regular, and column singular means not column regular. 
So much is standard. Some further definitions are needed in Section 3.

A partition $\lambda$ is strongly column regular if $\lambda \leq \mu$ for all column singular partitions $\mu$ in the same block as $\lambda$. For example $(4,3,1)>\left(4,2^{2}\right)>\left(4,1^{4}\right)$, yet for $p=3(4,3,1)$ is strongly column regular while $\left(4,2^{2}\right)$ is not, because of the separation into blocks.

A partition is row stable if its parts differ by at least $p-1$. This condition may be regarded as a strong form of row regularity: for $p=2$, it is the same condition, since either states that no two parts are equal.

A partition is horizontal if for each column of the diagram either all or none of the hook lengths is divisible by $p$. The reason for the name is that only 'horizontal' $p$-hooks, consisting of $p$ nodes lying in the same row, can be removed from such a diagram (see Remark 3.7). This condition may be regarded as a weak form of the Carter criterion, which states that the highest power of $p$ which divides the hook length is constant down each column. Clearly a horizontal partition must be row regular, and is column regular if and only if it is a core.

A partition is Steinberg if its adjacent parts differ by exactly $p-1$ and its smallest part is $<p$. These are precisely the partitions which are both row stable and column regular, and every Steinberg partition is a core. There are thus $p-1$ Steinberg partitions of given length $l>0$, which are the highest weights for the tensor products of the Steinberg module for $G L\left(l, F_{p}\right)$ by the determinant module.

\section{MODULAR SYMMETRIC FUNCTIONS}

Our discussion of modular symmetric functions will follow [4, §3], and we refer to [13, Chapter I] for background. Let $\Lambda_{n}$ be the ring of symmetric polynomials with integer coefficients in $n$ variables $x_{1}, \ldots, x_{n}$, graded as usual by the total degree $d \geq 0$. For each $n \geq 1$ we have an epimorphism $\Lambda_{n+1} \rightarrow \Lambda_{n}$ of graded rings, the projection, defined by $x_{i} \mapsto x_{i}$ for $i \leq n$ and $x_{n+1} \mapsto 0$. This is an isomorphism in degrees $d \leq n$. Let $\Lambda$ be the graded inverse limit of this sequence [13, I.2]. The elements of $\Lambda$ may be regarded as formal infinite sums of monomials in variables $x_{1}, x_{2}, \ldots$, and the projection $\Lambda \rightarrow \Lambda_{n}$ is an isomorphism in degrees $d \leq n$. We use the term 'symmetric function' for an element of $\Lambda$ or $\Lambda_{n}$.

For any integer $p \geq 2$, the modular complete symmetric function $h_{d}^{\prime}$ of degree $d \geq 0$ is defined by truncating the complete symmetric function $h_{d}$ at the $p$ th powers of the variables. More formally, in $\Lambda$ or $\Lambda_{n}$ let $m_{\lambda}$ be the monomial symmetric function associated with the partition $\lambda$. Then $h_{d}^{\prime}=\sum_{\lambda} m_{\lambda}$, where the sum is over all partitions $\lambda=\left(\lambda_{1}, \ldots, \lambda_{l}\right)$ of $d$ of length $l \leq n$ with $\lambda_{1}<p$. The functions $h_{d}^{\prime}$ are compatible with the projections, but in $\Lambda_{n}$, $h_{d}^{\prime}=0$ for $d>n(p-1)$. Of course, when $p=2, h_{d}^{\prime}=e_{d}$, the elementary symmetric function of degree $d$.

The classical Jacobi-Trudi identity [13, I (3.4)]

$$
s_{\lambda}=\operatorname{det}\left(h_{\lambda_{i}-i+j}\right), \quad 1 \leq i, j \leq l,
$$

defines the Schur function $s_{\lambda}$ in terms of complete symmetric functions. Here we make the usual convention that $h_{d}=0$ if $d<0$, and we also define $h_{d}^{\prime}=0$ for $d<0$. We define the modular Schur function $s_{\lambda}^{\prime}$ by the corresponding 
formula

$$
s_{\lambda}^{\prime}=\operatorname{det}\left(h_{\lambda_{i}-i+j}^{\prime}\right), \quad 1 \leq i, j \leq l .
$$

In particular, $s_{d}^{\prime}=h_{d}^{\prime}$. Once again, the special case $p=2$ does not give a 'new' symmetric function, because $s_{\lambda}^{\prime}=s_{\lambda^{\prime}}$, where $\lambda^{\prime}$ is the partition conjugate to $\lambda$ [13, I (3.5)].

As explained in $[4, \S 3.8]$, this idea may be extended to give modular analogues of any family of symmetric functions. Since $\Lambda=\mathbf{Z}\left[h_{1}, h_{2}, \ldots\right]$, and the $h_{d}$ are algebraically independent over $Z$ [13, I (2.8)], we may define for each integer $p \geq 2$ a ring homomorphism

$$
\omega^{\prime}: \Lambda \rightarrow \Lambda
$$

by the rule $\omega^{\prime}\left(h_{d}\right)=h_{d}^{\prime}$, for all $d \geq 0$. The image of a symmetric function under $\omega^{\prime}$ is then by definition the corresponding 'modular' symmetric function. (The reader who feels that this term presumes too much is assured that it is meant only to indicate dependence on the 'modulus' $p$. The alternative 'truncated symmetric function' would be misleading, as this behaviour of $h_{d}$ under $\omega^{\prime}$ is not typical of that of other symmetric functions.)

It follows from [4, Theorem 3.7] that $h_{1}^{\prime}, h_{2}^{\prime}, \ldots$ and $h_{1}^{\prime}, \ldots, h_{n}^{\prime}$ are algebraically independent in $\Lambda$ and $\Lambda_{n}$ respectively. (The proof given in [4] does not require the number of variables to be infinite.) Hence $\omega^{\prime}$ is injective for all $p \geq 2$. When $p=2, \omega^{\prime}=\omega$, the ring involution which interchanges the complete symmetric functions $h_{d}$ and the elementary symmetric functions $e_{d}$ [13, I, pp. 14-15]. For $p>2$, it follows from Proposition 1.2 below that $\omega^{\prime}$ is not surjective.

Remark 1.1. Since $\Lambda_{n}=\mathbf{Z}\left[h_{1}, \ldots, h_{n}\right]$, we can also define a ring homomorphism $\omega_{n}^{\prime}: \Lambda_{n} \rightarrow \Lambda_{n}$ by the rule $\omega_{n}^{\prime}\left(h_{d}\right)=h_{d}^{\prime}$, for $0 \leq d \leq n$. If we identify elements in $\Lambda$ and $\Lambda_{n}$ in degrees $\leq n$ using the projection, we have $\omega_{n}^{\prime}=\omega^{\prime}$ in these degrees. However, we do not use $\omega_{n}^{\prime}$ to define modular symmetric functions, because $\omega^{\prime}$ and $\omega_{n}^{\prime}$ do not commute with the projections $\Lambda \rightarrow \Lambda_{n}$ or $\Lambda_{n} \rightarrow \Lambda_{n-1}$. (For example, taking $p=2, \omega\left(e_{d}\right)=h_{d}$ for $n \geq d$, but in $\Lambda_{d-1}, e_{d}=0$ while $h_{d} \neq 0$; the formula for $e_{d}^{\prime}$ given in [4, Proposition $3.12(1)]$ shows that the situation when $p>2$ is similar.)

The power sums $P_{d}=\sum_{i} x_{i}^{d}$, where $d \geq 1$, play a central part in relating the classical symmetric functions to their modular analogues. The 'modular power sums' are defined by $P_{d}^{\prime}=\omega^{\prime}\left(P_{d}\right)$. The formula

$$
d h_{d}=\sum_{r=1}^{d} P_{r} h_{d-r}
$$

[13, I (2.11)] yields a corresponding formula

$$
d h_{d}^{\prime}=\sum_{r=1}^{d} P_{r}^{\prime} h_{d-r}^{\prime}
$$

which could alternatively be used to define them in terms of the $h_{d}^{\prime}$. The formula shows that $\Lambda \otimes_{\mathbf{z}} \mathbf{Q}=\mathbf{Q}\left[P_{1}^{\prime}, P_{2}^{\prime}, \ldots\right]$ and $\Lambda_{n} \otimes_{\mathbf{z}} \mathbf{Q}=\mathbf{Q}\left[P_{1}^{\prime}, \ldots, P_{n}^{\prime}\right]$.

Macdonald (see [4, Proposition 3.15(i)]) has made the following surprising observation, which generalises [13, I (2.13)]. 
Proposition 1.2. For any $n \geq 1, p \geq 2$ and $d \geq 0, P_{d}^{\prime}$ is related to $P_{d}$ by

$$
P_{d}^{\prime}= \begin{cases}P_{d}, & \text { if } d \not \equiv 0 \bmod p, \\ (1-p) P_{d}, & \text { if } d \equiv 0 \bmod p .\end{cases}
$$

It follows from this that $\omega^{\prime} \otimes_{\mathbf{Z}} \mathbf{Q}$ is a ring isomorphism, but that $\omega^{\prime}$ is not surjective in degrees $\geq p$, its eigenvalues being powers of $1-p$ [4, Proposition 3.15(iv)].

Remark 1.3. Another consequence of Proposition 1.2 is that the homomorphisms $\omega^{\prime}$ defined using any two moduli $p$ and $q$ commute. For example, from $\omega\left(h_{d}\right)=e_{d}$ we may deduce that $\omega\left(h_{d}^{\prime}\right)=e_{d}^{\prime}$, and, more generally, from $\omega\left(s_{\lambda}\right)=s_{\lambda^{\prime}}$ we have $\omega\left(s_{\lambda}^{\prime}\right)=s_{\lambda^{\prime}}^{\prime}$. Since $\omega$ is an isometry of $\Lambda$ with respect to the standard scalar product [13, I.4], it follows that $\left\langle s_{\lambda}^{\prime}, s_{\mu}\right\rangle=\left\langle s_{\lambda^{\prime}}^{\prime}, s_{\mu^{\prime}}\right\rangle$.

We next show that $[4$, Conjecture (2.6)] is equivalent to a statement about modular Schur functions. We recall that for given $n$ and $p$ this conjecture is shown in [4, Proposition 3.1] to be equivalent to the following statement.

Conjecture 1.4. The set $\left\{h_{\lambda}^{\prime} \mid l(\lambda) \leq n, \lambda_{1} \leq n(p-1)\right\}$ is linearly independent in $\Lambda_{n} \otimes \mathbf{Z} \mathbf{Q}$.

Recall that $h_{\lambda}=h_{\lambda_{1}} h_{\lambda_{2}} \cdots h_{\lambda_{l}}$ for the partition $\lambda=\left(\lambda_{1}, \lambda_{2}, \cdots, \lambda_{l}\right)$, so applying $\omega^{\prime}$ we have $h_{\lambda}^{\prime}=h_{\lambda_{1}}^{\prime} h_{\lambda_{2}}^{\prime} \cdots h_{\lambda_{l}}^{\prime}$. Since $h_{d}^{\prime}=0$ in $\Lambda_{n}$ when $d>$ $n(p-1), h_{\lambda}^{\prime}=0$ when $\lambda_{1}>n(p-1)$. In $\Lambda$, the elements $h_{\lambda}$ form a Z-basis indexed by the set of all partitions; hence the corresponding set of all $h_{\lambda}^{\prime}$ is a $\mathbf{Q}$-basis for $\Lambda \otimes_{\mathbf{Z}} \mathbf{Q}$. Since $\Lambda_{n}=\mathbf{Z}\left[h_{1}, h_{2}, \ldots, h_{n}\right]$, we see in the same way that $\left\{h_{\lambda}^{\prime} \mid \lambda_{1} \leq n\right\}$ is a $\mathbf{Q}$-basis for $\Lambda_{n} \otimes_{\mathbf{Z}} \mathbf{Q}$. Thus Conjecture 1.4 holds in degrees $d \leq n$ [4, Proposition 3.6]. In [4, §3.9] Conjecture 1.4 is proved in the case $n=2$. We prove it for $n=3$ in Section 2 (see Proposition 2.17).

Since $h_{d}^{\prime}=e_{d}$ for all $d$ if $p=2$, the conjecture is also true for all $n$ in this case. We may use this case $p=2$ to illustrate the next result. For $p=2$, conjugation of partitions exchanges the conditions $l(\lambda) \leq n$ and $\lambda_{1} \leq n(p-1)$, while $M\left(s^{\prime}, s\right)=J\left[13\right.$, p. 55], the matrix whose $(\lambda, \mu)$ entry is 1 if $\lambda^{\prime}=\mu$, and is 0 otherwise.

Let $M\left(s^{\prime}, s\right)=\left(m_{\lambda, \mu}\right)$ be the (infinite) transition matrix defined by $s_{\lambda}^{\prime}=$ $\sum_{\mu} m_{\lambda, \mu} s_{\mu}$, with rows and columns indexed by the set of all partitions. Since $\left\{s_{\lambda}\right\}$ is a $\mathbf{Z}$-basis of $\Lambda, m_{\lambda, \mu}$ is an integer, and since the basis $\left\{s_{\lambda}\right\}$ is orthonormal with respect to the standard scalar product on $\Lambda$ [13, I (4.8)], $m_{\lambda, \mu}=\left\langle s_{\lambda}^{\prime}, s_{\mu}\right\rangle$.

Proposition 1.5. For all $n \geq 1$ and $p \geq 2$, each of the following statements is equivalent to Conjecture 1.4 .

(i) The set $\left\{s_{\lambda}^{\prime} \mid l(\lambda) \leq n, \lambda_{1} \leq n(p-1)\right\}$ is linearly independent in $\Lambda_{n} \otimes_{\mathbf{Z}} \mathbf{Q}$.

(ii) The finite submatrix of $M\left(s^{\prime}, s\right)$ whose rows and columns are indexed by $\left\{\lambda \mid l(\lambda) \leq n, \lambda_{1} \leq n(p-1)\right\}$ is invertible over $\mathbf{Q}$.

Proof. We shall prove (i) by showing that $H_{n, p}^{\prime}=S_{n, p}^{\prime}$, where $H_{n, p}^{\prime}$ and $S_{n, p}^{\prime}$ denote the Q-spans of the functions $h_{\lambda}^{\prime}$ and $s_{\lambda}^{\prime}$, indexed in both cases by the set of partitions $\lambda$ such that $l(\lambda) \leq n$ and $\lambda_{1} \leq n(p-1)$. If $\lambda_{1}>n(p-1)$, $h_{\lambda}^{\prime}=0$ and hence, by the Jacobi-Trudi formula, $s_{\lambda}^{\prime}=0$ also. Hence $H_{n, p}^{\prime}$ and $S_{n, p}^{\prime}$ are unchanged if we replace the indexing set by the set of all $\lambda$ such that $l(\lambda) \leq n$. 
The (infinite) transition matrix $M(h, s)$, which expresses products of complete symmetric functions in terms of Schur functions, is the transpose of the Kostka matrix $K=K_{\lambda, \mu}[13,1.6]$. For any two partitions $\lambda$ and $\mu, K_{\lambda, \mu}$ is the number of semistandard tableaux of shape $\lambda$ and content $\mu$. In particular, $K_{\lambda, \mu}=0$ if $|\lambda| \neq|\mu|$. Applying $\omega^{\prime}$, this gives

$$
h_{\lambda}^{\prime}=\sum_{\mu \unrhd \lambda} K_{\mu, \lambda} s_{\mu}^{\prime}
$$

in $\Lambda$, and hence, by the projection $\Lambda \rightarrow \Lambda_{n}$, also in $\Lambda_{n}$. The condition $\mu \unrhd \lambda$ implies that $l(\mu) \leq l(\lambda)$, so the equation shows that $H_{n, p}^{\prime} \subseteq S_{n, p}^{\prime}$. The same argument applied to the inverse matrix $M\left(s^{\prime}, h^{\prime}\right)$ gives the reverse inequality $S_{n, p}^{\prime} \subseteq H_{n, p}^{\prime}$. This proves (i).

Next we shall prove that (ii) is equivalent to (i). For this, let $S_{n, p}$ and $M_{n, p}$ denote the Q-spans of the Schur functions $s_{\lambda}$ and the monomial symmetric functions $m_{\lambda}$, indexed in both cases by the set of partitions $\lambda$ such that $l(\lambda) \leq n$ and $\lambda_{1} \leq n(p-1)$. Since $s_{\lambda}=0$ and $m_{\lambda}=0$ if $l(\lambda)>n, S_{n, p}$ and $M_{n, p}$ are unchanged if we replace the indexing set by the set of all $\lambda$ such that $\lambda_{1} \leq n(p-1)$. We shall show that $S_{n, p}=M_{n, p}$.

The (infinite) transition matrix $M(s, m)$ is the Kostka matrix $K$. Thus

$$
s_{\lambda}=\sum_{\mu \unlhd \lambda} K_{\lambda, \mu} m_{\mu}
$$

in $\Lambda$, and hence, by the projection $\Lambda \rightarrow \Lambda_{n}$, also in $\Lambda_{n}$. The condition $\mu \unlhd \lambda$ implies that $\mu_{1} \leq \lambda_{1}$, so the equation shows that $S_{n, p} \subseteq M_{n, p}$. By applying the same argument to the inverse matrix $M(m, s)$, we obtain the reverse inequality $M_{n, p} \subseteq S_{n, p}$. Hence $S_{n, p}=M_{n, p}$.

We now observe that $H_{n, p}^{\prime} \subseteq M_{n, p}$. This is simply because, for $l(\lambda) \leq n$, $h_{\lambda}^{\prime}$ is the product of $\leq n$ polynomials, each having all their exponents $\leq p-1$. Hence every monomial appearing in $h_{\lambda}^{\prime}$ has all its exponents $\leq n(p-1)$.

To summarise, we have shown that $S_{n, p}^{\prime}=H_{n, p}^{\prime} \subseteq M_{n, p}=S_{n, p}$. Since the functions $m_{\lambda}$ and $s_{\lambda}$ are linearly independent, (i) holds if and only if $S_{n, p}^{\prime}=S_{n, p}$. This is equivalent to nonsingularity of the matrix defined in (ii).

A symmetry property of $M\left(s^{\prime}, s\right)$ with respect to conjugation was noted in Remark 1.3; we show in Corollary 1.7 below that this matrix is also symmetric in the usual sense.

Conjecture 1.4 can also be formulated in terms of the transition matrix $M\left(h^{\prime}, m\right)=\left(q_{\lambda, \mu}\right)$ [4, Proposition 3.4]. In its original form [4, Conjecture 2.6], the conjecture is restricted to the case where $p$ is prime. In this case we have a transition matrix $M\left(h^{\prime}, l\right)$, where $\left\{l_{\lambda}\right\}$ is the Z-basis of formal characters of simple polynomial representations of $G L(n, K)$ (see Section 4), and the conjecture states that the corresponding submatrix of this 'modular Kostka matrix' is nonsingular.

Since these families of symmetric functions are homogeneous, all these transition matrices are direct sums of matrices whose rows and columns are indexed by partitions of the same degree $d$. From now on, we shall use the notation $M\left(s^{\prime}, s\right)$ for one of these finite matrices. Thus, for $n \geq d, M\left(s^{\prime}, s\right)$ is indexed by the set of all partitions of $d$, and, in general, it is indexed by the 
set of all partitions $\lambda$ of degree $d$ such that $l(\lambda) \leq n$ and $\lambda_{1} \leq n(p-1)$. In other words, $M\left(s^{\prime}, s\right)$ is the matrix which represents the linear endomorphism $\omega^{\prime}$ of the Q-subspace of homogeneous polynomials of degree $d$ in $S_{n, p}$, with respect to the canonical basis of Schur functions.

Conjecture 1.4 states that $M\left(s^{\prime}, s\right)$ is invertible for all $p \geq 2, n \geq 1$, and $d \geq 0$. Some cases where the conjecture is known to be true were mentioned above. It is also trivially true if $d<p$, since $M\left(s^{\prime}, s\right)$ is the identity matrix. In Proposition 1.12 we extend this to the range $d<2 p$.

In Section 2, we shall prove a general 'block decomposition theorem' for $M\left(s^{\prime}, s\right)$ by combinatorial methods. For blocks of low weight, the same results can be obtained more directly by use of the Murnaghan-Nakayama formula [8, 2.4.7]. The method relies on Proposition 1.2 and the relation between symmetric functions and the ordinary character table of the symmetric group $S_{d}$ [13, p. 62].

We require some notation and basic formulas [8, Chapter $1 ; 13$, I.7]. For each partition $\rho$ of $d$, let $C(\rho)$ be the conjugacy class in $S_{d}$ consisting of all elements of cycle type $\rho$. Let $z_{\rho}$ be the order of the centralizer of an element of the class $C(\rho)$, so that $[13$, p. 17]

$$
z_{p}=\prod_{i \geq 1} i^{m_{i}} \cdot m_{i} !
$$

where $m_{i}$ is the number of parts of $\rho$ equal to $i$. For each partition $\lambda$ of $d$, let $\chi^{\lambda}$ be the ordinary irreducible character of $S_{d}$ corresponding to $\lambda$, and let $\chi_{\rho}^{\lambda}$ be its value on the class $C(\rho)$. Then [13, I (7.8)] the transition matrix $M(P, s)$ is the character table of $S_{d}$, i.e.

$$
P_{\rho}=\sum_{\lambda} \chi_{\rho}^{\lambda} s_{\lambda}
$$

and $M(s, P)=M(P, s)^{-1}$ is given by

$$
s_{\lambda}=\sum_{\rho} z_{\rho}^{-1} \chi_{\rho}^{\lambda} P_{\rho}
$$

We now relate this information to our matrix $M\left(s^{\prime}, s\right)$.

Proposition 1.6. For $n \geq d$, the columns of the (ordinary) character table of the symmetric group $S_{d}$ of degree $d$ form a complete set of orthogonal eigenvectors of $M\left(s^{\prime}, s\right)$.

Proof. By [4, Proposition 3.15], $M\left(s^{\prime}, s\right)$ is diagonalised by $M(P, s)$ when $n \geq d$, i.e.

$$
M(P, s) M\left(s^{\prime}, s\right) M(P, s)^{-1}=M\left(P^{\prime}, P\right) .
$$

By Proposition 1.2, the transition matrix $M\left(P^{\prime}, P\right)$ is diagonal, and the diagonal entry corresponding to $\rho$ is $(1-p)^{w(\rho)}$, where $w(\rho)$ is the number of parts of $\rho$ which are divisible by $p$.

It was noted above that the $(\rho, \lambda)$ entry of $M(P, s)$ is $\chi_{\rho}^{\lambda}$. It follows from the usual orthogonality relations for characters that the matrix $O=Z M(P, s)$ is orthogonal, where $Z$ is the diagonal matrix whose $\rho$ th entry is $z_{\rho}^{-1 / 2}$. Since $M\left(s^{\prime}, s\right)$ is diagonalised by $O$, it must be symmetric, and the rows of $O$ are eigenvectors of $M\left(s^{\prime}, s\right)$. More precisely, the column vector $\mathbf{a}_{\rho}$ whose $\lambda$ th entry is $\chi_{\rho}^{\lambda}$ lies in the $(1-p)^{w(\rho)}$-eigenspace of $M\left(s^{\prime}, s\right)$. 
Corollary 1.7. For all $p \geq 2, n \geq 1$, and $d \geq 0$, the matrix $M\left(s^{\prime}, s\right)$ is symmetric.

Proof. This has already been shown for $n \geq d$. For the general case we simply take the appropriate principal minor.

Recall $[8$, p. 241] that the conjugacy class $C(\rho)$ is called p-regular if its elements have order prime to $p$, i.e. if no part of $\rho$ is divisible by $p$, so that $w(\rho)=0$, and $p$-singular if $w(\rho)>0$. Hence $P_{\rho}^{\prime}=P_{\rho}$ if and only if the class $C(\rho)$ is $p$-regular.

Proposition 1.8. For all $p \geq 2$ and $n \geq 1, s_{\lambda}^{\prime}=s_{\lambda}$ if $\lambda$ is a core.

Proof. For any partition $\lambda$, by applying $\omega^{\prime}$ to the formula $s_{\lambda}=\sum_{\rho} z_{\rho}^{-1} \chi_{\rho}^{\lambda} P_{\rho}$, we have $s_{\lambda}^{\prime}=\sum_{\rho} z_{\rho}^{-1} \chi_{\rho}^{\lambda} P_{\rho}^{\prime}$. If $\lambda$ is a core, then $\chi_{\rho}^{\lambda}=0$ when $C(\rho)$ is $p$ singular. This follows from the Murnaghan-Nakayama formula [8, 2.4.7], since $k p$ is a part of $\rho$ for some positive integer $k$, while it is impossible to remove any $k p$-hook from $\lambda[8,2.7 .40]$. Both summations may therefore be taken over the set of $\rho$ for which $C(\rho)$ is $p$-regular. Since $P_{\rho}=P_{\rho}^{\prime}$ for all such $\rho$, $s_{\lambda}^{\prime}=s_{\lambda}$.

The next simplest case is a block of weight 1 . Recall that a partition $\lambda$ has weight 1 if it is reduced to its core $\nu$ by removal of a single $p$-hook, and that the sign $\varepsilon(\lambda)=(-1)^{h}$, where $h$ is the height of this $p$-hook.

Proposition 1.9. For all $p \geq 2, n \geq 1$

$$
s_{\lambda}^{\prime}=s_{\lambda}-\varepsilon(\lambda) P_{p} s_{\nu}
$$

when $\lambda$ is a partition of weight 1 with core $\nu$.

Proof. We apply $\omega^{\prime}$ to the formula

$$
s_{\lambda}=\sum_{|\rho|=d} z_{\rho}^{-1} \chi_{\rho}^{\lambda} P_{\rho},
$$

as in Proposition 1.8, and then subtract to obtain

$$
s_{\lambda}^{\prime}-s_{\lambda}=\sum_{|\rho|=d} z_{\rho}^{-1} \chi_{\rho}^{\lambda}\left(P_{\rho}^{\prime}-P_{\rho}\right) .
$$

If $C(\rho)$ is $p$-regular, then $P_{\rho}^{\prime}=P_{\rho}$. If $\rho$ has a part divisible by $p$ and $>p$, then $\chi_{\rho}^{\lambda}=0$ by the Murnaghan-Nakayama formula, since we cannot remove a hook of the corresponding length from the diagram of $\lambda$. A similar argument, applying the Murnaghan-Nakayama formula twice, shows that $\chi_{\rho}^{\lambda}=0$ if $\rho$ has more than one part equal to $p$.

Hence the only partitions $\rho$ which contribute to the above sum are those with one part equal to $p$, while the remaining parts form a partition $\sigma$ of $d-p$ such that $C(\sigma)$ is a $p$-regular conjugacy class in $S_{n-p}$. We then have $z_{\rho}=p z_{\sigma}$, $P_{\rho}^{\prime}=(1-p) P_{\rho}$, and $P_{\rho}=P_{p} P_{\sigma}$. The Murnaghan-Nakayama formula gives

$$
\chi_{\rho}^{\lambda}=-\varepsilon(\lambda) \chi_{\sigma}^{\nu},
$$

the minus sign occurring because the height of a hook is one less than its 'leg length'. Hence

$$
s_{\lambda}^{\prime}-s_{\lambda}=\varepsilon(\lambda) P_{p} \sum_{\sigma} z_{\sigma}^{-1} \chi_{\sigma}^{\nu} P_{\sigma},
$$


where the sum is over all $p$-regular conjugacy classes in $S_{d-p}$. Since $\nu$ is a core, it follows from the proof of Proposition 1.8 that this sum is equal to $s_{\nu}$.

The same method may be applied in principle to expand $s_{\lambda}^{\prime}$ in terms of products of the form $P_{p \alpha} s_{\beta}$ when the partition $\lambda$ has weight $w>1$. However, one has to keep track of all possible sequences of removals of hooks of length divisible by $p$. This is not practicable except in special cases, and even in these cases the formulas obtained do not appear to be efficient.

We can use Proposition 1.9 to calculate the entries of the matrices $M\left(s^{\prime}, s\right)$ which correspond to partitions of weight 1 .

Corollary 1.10. Let $p \geq 2, n \geq 1$ and let $\lambda$ be a partition of weight 1 with core $\nu$. Then if $\mu$ has weight 1 and core $\nu$, and $\mu \neq \lambda$,

$$
\left\langle s_{\lambda}^{\prime}, s_{\mu}\right\rangle=-\varepsilon(\lambda) \varepsilon(\mu)
$$

and otherwise $\left\langle s_{\lambda}^{\prime}, s_{\mu}\right\rangle=0$.

Proof. By [13, I.3 Example 11],

$$
P_{p} s_{\nu}=\sum_{\mu}(-1)^{h(\mu)} s_{\mu},
$$

where the sum is over all partitions $\mu$ which can be obtained from $\nu$ by adding a $p$-hook, and $h(\mu)$ is the height of this $p$-hook. Since $\varepsilon(\mu)=(-1)^{h(\mu)}$, by Proposition 1.9 we have

$$
s_{\lambda}^{\prime}=s_{\lambda}-\varepsilon(\lambda) \sum_{\mu} \varepsilon(\mu) s_{\mu}=-\varepsilon(\lambda) \sum_{\mu \neq \lambda} \varepsilon(\mu) s_{\mu} .
$$

The result follows by orthonormality of $\left\{s_{\mu}\right\}$.

Proposition 1.8 and Corollary 1.10 show that the matrices $M\left(s^{\prime}, s\right)$ have direct summands corresponding to blocks of weight 0 (cores) and weight 1 . In the next section we shall extend this to blocks of arbitrary weight. Obviously blocks of weight $\geq 2$ occur only for $d \geq 2 p$, so the results proved so far suffice to prove Conjecture 1.4 for $d<2 p$.

Example 1.11. In the case $n=p=d=5$, there are two 5-cores, $(3,2)$ and $(2,2,1)$, and a block of weight 1 consisting of the partitions $\left(1^{5}\right),\left(2,1^{3}\right)$, $\left(3,1^{2}\right),(4,1)$, and $(5)$. Using this lexicographic order, the transition matrix $M\left(s^{\prime}, s\right)$ for the block is

$$
A=\left(\begin{array}{rrrrr}
0 & 1 & -1 & 1 & -1 \\
1 & 0 & 1 & -1 & 1 \\
-1 & 1 & 0 & 1 & -1 \\
1 & -1 & 1 & 0 & 1 \\
-1 & 1 & -1 & 1 & 0
\end{array}\right)
$$

We have $A=I-\mathbf{a a}^{\top}$, where $\mathbf{a}^{\top}$ is the row vector $(1,-1,1,-1,1)$.

This example is typical for a block of weight 1 . In general, such a block contains $p$ partitions, since there is precisely one way to add a $p$-hook of given height to any core $[8, \S 2.7]$. Thus the signs alternate, and the transition matrix is $A=I-\mathbf{a a}^{\top}$, where a is the column vector whose $k$ th entry is $(-1)^{k-1}$, $1 \leq j \leq p$. By Proposition 1.2, or an easy calculation, the eigenvalues of $A$ are 1 (with multiplicity $p-1$ ) and $1-p$. 
Recall that the rows and columns of $M\left(s^{\prime}, s\right)$ are indexed by the partitions $\lambda$ of $d$ such that $l(\lambda) \leq n$ and $\lambda_{1} \leq n(p-1)$. For example, when $p=d=5$ and $n=2$, only $(5),(3,2)$, and $(4,1)$ qualify, and we must consider a $2 \times 2$ submatrix of the matrix $A$ of Example 1.11. It is easy to see that the required submatrix always has the same form as $A$, but for a possibly smaller value of $p$.

To complete the proof of Conjecture 1.4 in the range $d<2 p$, we need therefore only show that the case of a $1 \times 1$ submatrix cannot arise. In other words, we must show that for all $p \geq 2, n \geq 1$, and for all cores $\nu$, there cannot be exactly one way to add a $p$-hook to a core $\nu$ to give a partition $\lambda$ such that $l(\lambda) \leq n$ and $\lambda_{1} \leq n(p-1)$.

This is easy to see using the abacus representation of partitions. The condition $l(\lambda) \leq n$ implies that we need only consider partitions which can be represented on the abacus by a set of $n$ beads. The condition $\lambda_{1} \leq n(p-1)$ implies that with $n$ beads the highest permissible bead position is $n(p-1)+n-1=$ $n p-1$; i.e. all the beads lie on the top $n$ rows of the abacus. Thus if all the beads for the core $\nu$ lie on the same runner, none of the weight 1 partitions qualify. In all other cases, there must be at least two beads in the representation of $\nu$ which can be moved down one place on the corresponding runner without leaving the top $n$ rows. The corresponding partitions give a matrix of the same form as $A$ which is nonsingular.

We have proved

Proposition 1.12. Conjecture 1.4 is true for all $n$ in the range $d<2 p$.

\section{BLOCKS AND QUOTIENTS}

In this section we shall obtain general formulae which express modular Schur functions in terms of ordinary Schur functions. All the results in this section are valid for any modulus $p \geq 2$.

We begin by defining a ring homomorphism $\psi_{p}: \Lambda \rightarrow \Lambda$, called the $p$ quotient. This definition is based on the work of Littlewood [11, 12]; when the core of $\lambda$ is $(0), \psi_{p}\left(s_{\lambda}\right)$ is the $p$-quotient of $\lambda$ in the sense of [12], but, in the general case, when the core is $\mu$, Littlewood's $p$-quotient is the function $\psi_{p}\left(s_{\lambda / \mu}\right)$ of Proposition 2.3.

As in the case of $\omega^{\prime}$, we define $\psi_{p}$ as a ring homomorphism by specifying its value on the complete symmetric functions $h_{d}$, by the rule

$$
\psi_{p}\left(h_{d}\right)= \begin{cases}h_{d / p}, & \text { if } p \text { divides } d \\ 0, & \text { otherwise. }\end{cases}
$$

Proposition 2.1. If some part of $\mu$ is not divisible by $p$, then

$$
\psi_{p}\left(h_{\mu}\right)=\psi_{p}\left(e_{\mu}\right)=\psi_{p}\left(P_{\mu}\right)=0 .
$$

Otherwise, let $\mu=p \lambda$. Then

$$
\psi_{p}\left(h_{\mu}\right)=h_{\lambda}, \quad \psi_{p}\left(e_{\mu}\right)=(-1)^{(p-1)|\lambda|} e_{\lambda}, \quad \psi_{p}\left(P_{\mu}\right)=p^{l(\lambda)} P_{\lambda} .
$$

Proof. Let $H(t)=\sum_{d \geq 0} h_{d} t^{d}, E(t)=\sum_{d \geq 0} e_{d} t^{d}$, and $P(t)=\sum_{d \geq 0} P_{d} t^{d}$ denote the generating functions for the complete symmetric functions $h_{d}$, the elementary symmetric functions $e_{d}$, and the power sums $P_{d}$. 
From the definition of $\psi_{p}$, we have $\psi_{p}(H(t))=H\left(t^{p}\right)$. Since $H(t) E(-t)=1$ $[13, \mathrm{I}(2.6)]$, it follows that $\psi_{p} E(-t)=E\left(-t^{p}\right)$.

Similarly the equation $P(t)=H^{\prime}(t) / H(t)$ [13, I (2.10)], where $H^{\prime}(t)$ denotes the derivative $d H(t) / d t$, gives $\psi_{p}(P(t))=\psi_{p}\left(H^{\prime}(t)\right) / \psi_{p}(H(t))=p H^{\prime}\left(t^{p}\right) / H\left(t^{p}\right)$ $=p P\left(t^{p}\right)$. The results follow by equating powers of $t$ and using the fact that $\psi_{p}$ is a ring homomorphism.

Recall the $p$ th power (or Frobenius) map $\psi^{p}: \Lambda \rightarrow \Lambda$, which is the ring homomorphism defined by replacing each variable $x_{i}$ in $f$ by $x_{i}^{p}$, and regarding the result as a symmetric function of the $x_{i}$. (Often, $\psi^{p}(f)$ is written as $f^{(F)}$; the present notation is chosen to fit with Adams operations, cf. [13, p. 18].) The operations $\psi_{p}$ and $\psi^{p}$ are adjoint with respect to the standard scalar product on $\Lambda$, i.e.

Proposition 2.2. For all symmetric functions $f, g \in \Lambda$,

$$
\left\langle\psi^{p}(f), g\right\rangle=\left\langle f, \psi_{p}(g)\right\rangle .
$$

Proof. By [13, I (4.7)], the $P_{\lambda}$ form an orthogonal basis of $\Lambda$, and $\left\langle P_{\lambda}, P_{\lambda}\right\rangle=$ $z_{\lambda}$. Since $z_{p \lambda}=p^{l(\lambda)} z_{\lambda}$, we have $\left\langle\psi^{p}\left(P_{\lambda}\right), P_{\mu}\right\rangle=\delta_{p \lambda, \mu} p^{l(\lambda)} z_{\lambda}=\left\langle P_{\lambda}, \psi_{p}\left(P_{\mu}\right)\right\rangle$. Since $P_{\lambda}$ is a $\mathbf{Q}$-basis of $\Lambda \otimes_{\mathbf{Z}} \mathbf{Q}$, the result follows by linearity.

Littlewood's definition of $\psi_{p}[11, \S 7.3]$ explains this adjoint relationship. Given a symmetric function $f$ in the variables $x_{1}, x_{2}, \ldots$, let $g$ be the corresponding symmetric function in the variables $\zeta^{j} x_{i}$, where $\zeta=e^{2 \pi i / p}$ and $0 \leq j \leq p-1$. In fact, $g$ is a polynomial in $x_{1}^{p}, x_{2}^{p}, \ldots$, and the function obtained by replacing each $x_{i}^{p}$ in $g$ by $x_{i}$ is $\psi_{p}(f)$. This can be seen by performing the calculation for the special case $f=P_{d}$, using the fact that $\zeta, \zeta^{2}, \ldots, \zeta^{p-1}$ are the roots of the equation $1+z+z^{2}+\cdots+z^{p-1}=0$, and then using the obvious multiplicativity and linearity properties to extend over $\Lambda \otimes \mathbf{Z} \mathbf{Q}$.

In order to evaluate $\psi_{p}$ on Schur functions, it is convenient to deal with the more general class of skew Schur functions $s_{\lambda / \mu}$. For any two partitions $\lambda$ and $\mu$, this function may be defined by the generalised Jacobi-Trudi formula [13, I (5.4)]

$$
s_{\lambda / \mu}=\operatorname{det}\left(h_{\lambda_{i}-\mu_{j}-i+j}\right),
$$

where as usual $\lambda_{i}=0$ if $i>l(\lambda)$, and similarly for $\mu$. Thus $s_{\lambda /(0)}=s_{\lambda}$. It is easy to see that $s_{\lambda / \mu}=0$ unless $\lambda_{i} \geq \mu_{i}$ for all $i$, and that in this case it depends only on the skew diagram obtained by removing the Young diagram of $\mu$ from the north-west corner of the diagram of $\lambda$. We shall denote this skew diagram by $\lambda / \mu$ (this is written as $\lambda-\mu$ in [13]); of course $\lambda /(0)=\lambda$.

For the next result, we recall from Section 0 the notation $\varepsilon(\lambda)$ for the sign of the partition $\lambda$, and the notation $(\lambda(0), \lambda(1), \ldots, \lambda(p-1))$ for the quotient of $\lambda$.

Proposition 2.3. If $\lambda$ and $\mu$ have the same core, then

$$
\psi_{p}\left(s_{\lambda / \mu}\right)=\varepsilon(\lambda) \varepsilon(\mu) \prod_{k=0}^{p-1} s_{\lambda(k) / \mu(k)},
$$

and otherwise $\psi_{p}\left(s_{\lambda / \mu}\right)=0$.

Proof. Take sequences of $\beta$-numbers for $\lambda$ and $\mu$, of the same length $b$. The choice of $b$ makes no difference to the argument, and we may assume, by 
adding zero parts to $\lambda$ and $\mu$ if necessary, that $b$ is the size of the Jacobi-Trudi determinant for $s_{\lambda / \mu}$. Since $\psi_{p}$ is a ring homomorphism, it follows that

$$
\psi_{p}\left(s_{\lambda / \mu}\right)=\operatorname{det}\left(\psi_{p}\left(h_{\lambda_{i}-\mu_{j}-i+j}\right)\right) \text {. }
$$

By Proposition 2.1, the $(i, j)$ th entry is nonzero if and only if $\lambda_{i}-i \equiv \mu_{j}-j$ $\bmod p$; i.e. the $i$ th $\beta$-number of $\lambda$ is congruent to the $j$ th $\beta$-number of $\mu$. Thus the nonzero entries form $p$ submatrices, the $k$ th submatrix being defined by the rows $i$ and the columns $j$ such that the $i$ th $\beta$-number of $\lambda$ and the $j$ th $\beta$-number of $\mu$ are congruent to $k \bmod p$.

Let us permute the two sequences of $\beta$-numbers by sorting them into residue classes $\bmod p$, taken in the order $k=0,1, \ldots, p-1$, while keeping the decreasing order within each residue class. Suppose that, for $\lambda$, the subsequence of $\beta$-numbers congruent to $k \bmod p$ is $\left(a_{1} p+k, a_{2} p+k, \ldots, a_{r(k)} p+k\right)$, and that, for $\mu$, it is $\left(b_{1} p+k, b_{2} p+k, \ldots, b_{s(k)} p+k\right)$.

If we apply the same two permutations to the rows and columns of the determinant, then it takes the form

$$
\left|\begin{array}{cccc}
A(0) & 0 & \ldots & 0 \\
0 & A(1) & \ldots & 0 \\
\vdots & \vdots & \ddots & \vdots \\
0 & 0 & \ldots & A(p-1)
\end{array}\right|
$$

where $A(k)$ is an $r(k) \times s(k)$ matrix. By elementary linear algebra, this determinant is zero unless $r(k)=s(k)$ for $0 \leq k \leq p-1$. This condition means that the abacus representations of $\lambda$ and $\mu$ have the same number of beads on each runner, i.e. $\lambda$ and $\mu$ have the same core.

Thus we may assume $r(k)=s(k)=r$, say. Since the sequences $\left(a_{1} p+k, a_{2} p+k, \ldots, a_{r} p+k\right)$ and $\left(b_{1} p+k, b_{2} p+k, \ldots, b_{r} p+k\right)$ are strictly decreasing, the sequences $\lambda(k)=\left(a_{1}-r+1, a_{2}-r+2, \ldots, a_{r}\right)$ and $\mu(k)=\left(b_{1}-r+1, b_{2}-r+2, \ldots, b_{r}\right)$ are partitions, of length $\leq r$, and $\left(a_{1}, a_{2}, \ldots, a_{r}\right),\left(b_{1}, b_{2}, \ldots, b_{r}\right)$ are sequences of $\beta$-numbers for $\lambda(k)$ and $\mu(k)$ respectively.

Now the $(u, v)$ th entry of the matrix $A(k)$ is $h_{d}$, where

$$
d=\left(\left(a_{u} p+k\right)-\left(b_{v} p+k\right)\right) / p=a_{u}-b_{v} .
$$

Since this is the difference of the $u$ th $\beta$-number of $\lambda(k)$ and the $v$ th $\beta$-number of $\mu(k), \operatorname{det}(A(k))$ is the Jacobi-Trudi determinant for $s_{\lambda(k) / \mu(k)}$.

Finally, we must check that the row and column permutations we have used multiply the determinant by $\varepsilon(\lambda) \varepsilon(\mu)$. To see this, carry out the permutations of $\beta$-numbers in two steps, first sorting the beads into the order in which they appear in the abacus representation of the core. This introduces signs $\varepsilon(\lambda)$ for the row permutation and $\varepsilon(\mu)$ for the column permutation. The second step, which reads the abacus in 'transpose' order, is the same for both rows and columns, and so it does not affect the sign.

Example 2.4. In Corollary 1.10, we used a special case of the formula of [13, I.3, Example 11] for the product of a Schur function by the power sum $P^{p}$. This formula (in the general case) can be derived as follows: $\left\langle P_{p} s_{\mu}, s_{\lambda}\right\rangle=$ $\left\langle\psi^{p}\left(s_{1}\right) s_{\mu}, s_{\lambda}\right\rangle=\left\langle\psi^{p}\left(s_{1}\right), s_{\lambda / \mu}\right\rangle$ (by [13, I (5.1)]) $=\left\langle s_{1}, \psi_{p}\left(s_{\lambda / \mu}\right)\right\rangle$ (by Proposition 2.2); now, by Proposition 2.3, $\psi_{p}\left(s_{\lambda / \mu}\right)=(-1)^{h} s_{1}$ if and only if $\lambda / \mu$ is 
a skew $p$-hook of height $h=h(\lambda / \mu)$. Hence $P_{p} s_{\mu}=\sum_{\lambda}(-1)^{h(\lambda / \mu)} s_{\lambda}$, summed over all such partitions $\lambda$.

We now turn to our main objective in this section, namely the evaluation of modular Schur functions in terms of ordinary ones. The skew Schur functions play an intermediate part in this process. The next result is the major step in the proof that $s_{\lambda}^{\prime}$ is an integer combination of the functions $s_{\mu}$ for partitions $\mu$ which have the same core as $\lambda$. This generalises the results proved in the previous section for cores and blocks of weight 1 .

Theorem 2.5. For any partition $\lambda$,

$$
s_{\lambda}^{\prime}=\sum_{\nu}(-1)^{|\lambda|-|\nu|} \psi^{p}\left(\psi_{p}\left(s_{\lambda^{\prime} / \nu^{\prime}}\right)\right) s_{\nu} .
$$

Remark 2.6. This formula, and its proof, can be generalised to give an expansion of $s_{\lambda / \mu}^{\prime}$ for any partition $\mu$ : we need only replace the factor $s_{\nu}$ on the right by $s_{\nu / \mu}$.

Proof of Theorem 2.5. First note that this formal sum over all partitions $\nu$ is finite, since $s_{\lambda^{\prime} / \nu^{\prime}}=0$ unless the diagram of $\nu$ is a subset of the diagram of $\lambda$.

The case $\lambda=(d)$ is proved in [4, Proposition 3.12]. In this case, the formula is

$$
h_{d}^{\prime}=\sum_{d=p q+r}(-1)^{q} \psi^{p}\left(e_{q}\right) h_{r},
$$

which follows from the observation that the generating function $H^{\prime}(t)=$ $\sum_{d \geq 0} h_{d}^{\prime} t^{d}$ is given by

$$
H^{\prime}(t)=\prod_{i \geq 1} \frac{1-x_{i}^{p} t^{p}}{1-x_{i} t}=\psi^{p} E\left(-t^{p}\right) H(t) .
$$

Here it is convenient to let $q$ and $r$ run over all integer values, with the usual convention that $h_{d}=e_{d}=0$ for $d<0$.

We use this formula to expand all the entries in the Jacobi-Trudi determinant

$$
s_{\lambda}^{\prime}=\operatorname{det}\left(h_{\lambda_{i}-i+j}^{\prime}\right), \quad 1 \leq i, j \leq l=l(\lambda) .
$$

Each row of the determinant may now be regarded as a sum of row vectors corresponding to the values of $q$ appearing in that row. Using the multilinearity of the determinant in its rows, we may write the determinant as a sum of determinants with $(i, j)$ th entries of the form $(-1)^{q} \psi^{p}\left(e_{q}\right) h_{r}$, where $p q+r=$ $\lambda_{i}-i+j$ and $q$ is constant along each row. Hence

$$
s_{\lambda}^{\prime}=\sum_{\alpha}(-1)^{|\alpha|} \psi^{p}\left(e_{\alpha}\right) \operatorname{det}\left(h_{\beta_{i}-i+j}\right),
$$

where $\alpha=\left(\alpha_{1}, \ldots, \alpha_{l}\right)$ and $\beta=\left(\beta_{1}, \ldots, \beta_{l}\right)$ run over all $l$-tuples of integers such that $p \alpha_{i}+\beta_{i}=\lambda_{i}$, and $|\alpha|=\sum_{i} \alpha_{i}=q$, and $|\beta|=\sum_{i} \beta_{i}=r$. (Here, as usual, we denote the product $e_{\alpha_{1}} \cdots e_{\alpha_{l}}$ by $e_{\alpha}$, although $\alpha$ need not be a partition.)

In the case where $\beta$ is a partition $\nu$, the determinant $\operatorname{det}\left(h_{\beta_{i}-i+j}\right)$ is the Jacobi-Trudi determinant for $s_{\nu}$. In the general case, consider the set of integers $\beta_{i}-i+l, 1 \leq i \leq l$. If any of these numbers is negative, the determinant has a zero row, and if any two of the numbers are equal, it has two equal rows. 
Thus if the determinant is nonzero, these numbers are a set of $\beta$-numbers for a partition $\nu=\left(\nu_{1}, \ldots, \nu_{l}\right)$ of degree $|\nu|=r$, and

$$
\operatorname{det}\left(h_{\beta_{i}-i+j}\right)=(-1)^{\operatorname{sgn}(\pi)} s_{\nu}
$$

where $\pi$ is the permutation of the suffixes $1,2, \ldots, l$ which puts the numbers $\beta_{i}-i$ in strictly decreasing order.

We next collect the terms in the sum which correspond in this way to the same partition $\nu$. For each $\nu$, we have to sum over all permutations of $1,2, \ldots, l$. Given $\nu$ and a permutation $\pi \in S_{l}$, we define a corresponding $l$-tuple of integers $\alpha(\pi)=\left(\alpha(\pi)_{1}, \ldots, \alpha(\pi)_{l}\right)$ by the rule

$$
\nu_{j}-j+l=\lambda_{i}-i-p \alpha(\pi)_{i}+l,
$$

where $i=\pi(j)$ for $1 \leq j \leq l$.

Thus $|\alpha(\pi)|=q$ for all $\pi$, where $p q=|\lambda|-|\nu|$. Then the above sum for $s_{\lambda}^{\prime}$ can be rewritten as

$$
s_{\lambda}^{\prime}=\sum_{\nu}(-1)^{q} \psi^{p}\left(f_{\nu}\right) s_{\nu},
$$

where the sum is over all partitions $\nu$ of length $\leq l$, and

$$
f_{\nu}=\sum_{\pi \in S_{l}}(-1)^{\operatorname{sgn}(\pi)} e_{\alpha(\pi)} .
$$

Now by Proposition 2.1

and hence

$$
e_{\alpha(\pi)}=(-1)^{(p-1) q} \psi_{p}\left(e_{p \alpha(\pi)}\right)
$$

by $[13, \mathrm{I}(5.5)]$.

$$
\begin{aligned}
f_{\nu} & =(-1)^{(p-1) q} \sum_{\pi \in S_{l}}(-1)^{\operatorname{sgn}(\pi)} \psi_{p}\left(e_{p \alpha(\pi)}\right) \\
& =(-1)^{(p-1) q} \psi_{p}\left(\sum_{\pi \in S_{l}}(-1)^{\operatorname{sgn}(\pi)} e_{p \alpha(\pi)}\right) \\
& =(-1)^{(p-1) q} \psi_{p}\left(\operatorname{det}\left(e_{\lambda_{i}-\nu_{j}-i+j}\right)\right) \\
& =(-1)^{(p-1) q} \psi_{p}\left(S_{\lambda^{\prime} / \nu^{\prime}}\right)
\end{aligned}
$$

The proof of Theorem 2.5 is completed by substituting this expression for $f_{\nu}$ in the main sum, noting that $|\lambda|-|\nu|=p q$.

Proposition 2.7. For all partitions $\lambda$ and $\mu$,

$$
\left\langle s_{\lambda}^{\prime}, s_{\mu}\right\rangle=\sum_{\nu}(-1)^{|\lambda|-|\nu|}\left\langle\psi_{p}\left(s_{\lambda^{\prime} / \nu^{\prime}}\right), \psi_{p}\left(s_{\mu / \nu}\right)\right\rangle .
$$

Proof. For all partitions $\lambda, \mu$, and $\nu,\left\langle s_{\lambda} s_{\nu}, s_{\mu}\right\rangle=\left\langle s_{\lambda}, s_{\mu / \nu}\right\rangle[13, \mathrm{I}(5.1)]$. Since the functions $s_{\lambda}$ are a Z-basis for $\Lambda$, if follows that $\left\langle f s_{\nu}, s_{\mu}\right\rangle=\left\langle f, s_{\mu / \nu}\right\rangle$ for all $f \in \Lambda$. Hence by Theorem 2.5

$$
\begin{aligned}
\left\langle s_{\lambda}^{\prime}, s_{\mu}\right\rangle & =\sum_{\nu}(-1)^{|\lambda|-|\nu|}\left\langle\psi^{p}\left(\psi_{p}\left(s_{\lambda^{\prime} / \nu^{\prime}}\right)\right) s_{\nu}, s_{\mu}\right\rangle \\
& =\sum_{\nu}(-1)^{|\lambda|-|\nu|}\left\langle\psi^{p}\left(\psi_{p}\left(s_{\lambda^{\prime} / \nu^{\prime}}\right)\right), s_{\mu / \nu}\right\rangle
\end{aligned}
$$

from which the result follows by Proposition 2.2. 
Corollary 2.8. The transition matrix $M\left(s^{\prime}, s\right)$ is the direct sum of submatrices indexed by the cores of partitions of degree $d$; i.e. $\left\langle s_{\lambda}^{\prime}, s_{\mu}\right\rangle=0$ unless $\lambda$ and $\mu$ are in the same block.

Proof. By Proposition 2.3, $\psi_{p}\left(s_{\lambda^{\prime} / \nu^{\prime}}\right)=0$ unless $\lambda$ and $\nu$ have the same core, and $\psi_{p}\left(s_{\mu / \nu}\right)=0$ unless $\mu$ and $\nu$ have the same core. Thus the sum in Proposition 2.7 is 0 unless $\lambda$ and $\mu$ have the same core.

By Proposition 2.3 and the definition of skew Schur functions, the sum in Proposition 2.7 may be taken over partitions $\nu$ such that $\lambda(k)_{i} \geq \nu(k)_{i}$ and $\mu(k)_{i} \geq \nu(k)_{i}$ for $k=0,1, \ldots, p-1$ and all $i$. In terms of Young diagrams, this condition means that the diagram of $\nu$ is contained in the intersection of the diagrams of $\lambda$ and $\mu$, and can be obtained from either of these diagrams by removal of suitable sequences of $p$-hooks.

The following alternative form of Proposition 2.7 shows that $\left\langle s_{\lambda}^{\prime}, s_{\mu}\right\rangle$ is determined by the signs and quotients of $\lambda$ and $\mu$.

Proposition 2.9. Let $\lambda$ and $\mu$ be partitions with weight $w$. Then

$$
\left\langle s_{\lambda}^{\prime}, s_{\mu}\right\rangle=(-1)^{w} \varepsilon(\lambda) \varepsilon(\mu) \sum_{\nu}(-1)^{w(\nu)}\left\langle\prod_{k=0}^{p-1} s_{\lambda^{\prime}(k) / \nu^{\prime}(k)}, \prod_{k=0}^{p-1} s_{\mu(k) / \nu(k)}\right\rangle .
$$

Proof. This follows on substituting in Proposition 2.7 the expressions for $\psi_{p}\left(s_{\lambda^{\prime} / \nu^{\prime}}\right)$ and $\psi_{p}\left(s_{\mu / \nu}\right)$ given by Proposition 2.3, and using the formula $\varepsilon\left(\lambda^{\prime}\right)=$ $(-1)^{(p-1) w} \varepsilon(\lambda)$ (and similarly for $\mu$ ) of Section 0 to simplify the signs.

The sum in Proposition 2.9 is completely determined by the partitions $\lambda(i)$ and $\mu(i)$ for $0 \leq i \leq p-1$, and is unchanged by permuting the indices $i$. We reformulate this observation as follows.

Corollary 2.10. Let $\lambda$ and $\mu$ be partitions in the same block, of weight $w$, and let $a_{\lambda, \mu}=(-1)^{w} \varepsilon(\lambda) \varepsilon(\mu)\left\langle s_{\lambda}^{\prime}, s_{\mu}\right\rangle$. Then $a_{\lambda, \mu}$ depends only on the quotients of $\lambda$ and $\mu$, and not on their core. More precisely, it depends only on the set of ordered pairs of partitions $(\lambda(i), \mu(i)), 0 \leq i \leq p-1$.

Suppose that for given $p$ and $w$ we choose a fixed ordering on the set of all $p$-tuples of partitions of total degree $w$. For example, there are $p$ such quotients if $w=1$, and they have a natural ordering (cf. Example 1.11), but in general there seems to be no preferred choice. We then have a matrix $A=\left(a_{i, j}\right)$ depending only on $p$ and $w$, where $a_{i, j}=a_{\lambda, \mu}$, and $\lambda$ and $\mu$ are those partitions, in any block of weight $w$, whose $p$-quotients are the $i$ th and $j$ th respectively in the chosen ordering. For example, when $w=1$ and the 'natural' ordering of quotients is used, $a_{i, j}=0$ if $i=j$ and $a_{i, j}=1$ if $i \neq j$.

Clearly, any values of $i$ for which both partitions $\lambda(i)$ and $\mu(i)$ are $(0)$ do not affect the value of $a_{\lambda, \mu}$. Thus the effect of choosing a smaller modulus $p$ is to extract a submatrix of $A$, and given $w$, the entries in $A$ for all values of $p$ are entries in $A$ for $p=2 w$. For $p=2 w$, if $\lambda(i)+\mu(i)=(1)$ for all $i$ then the sum in Proposition 2.9 is easily evaluated: there is only one term, corresponding to the core $\nu$, and so $a_{\lambda, \mu}=\left\langle s_{1}^{w}, s_{1}^{w}\right\rangle=\left\langle P_{1}^{w}, P_{1}^{w}\right\rangle=\left\langle P_{\left(1^{w}\right)}, P_{\left(1^{w}\right)}\right\rangle=z_{\left(1^{w}\right)}=w !$. We conjecture that all the entries of $A$ lie in the range $0 \leq a_{\lambda, \mu} \leq w$ !.

We can use Proposition 2.9 to detect some zero entries in the transition matrix $M\left(s^{\prime}, s\right)$ for a block with any core and weight. 
Lemma 2.11. If the quotient of $\lambda$ has rth component of length $l$ and all other components are (0), and if, for some $s \neq r$, the sth component of the quotient of $\mu$ has a part $>l$, then $\left\langle s_{\lambda}^{\prime}, s_{\mu}\right\rangle=0$.

Proof. In Proposition 2.7, we need only consider partitions $\nu$ with $\nu(k)=(0)$ for $k \neq r$. Proposition 2.3 now gives $\psi_{p}\left(s_{\lambda^{\prime} / \nu^{\prime}}\right)=\varepsilon\left(\lambda^{\prime}\right) \varepsilon\left(\nu^{\prime}\right) s_{\lambda(r)^{\prime} / \nu(r)^{\prime}}$. Since $\lambda(r)_{1}^{\prime}=l(\lambda(r))=l, s_{\lambda(r)^{\prime} / \nu(r)^{\prime}}$ can be expanded, using the Littlewood-Richardson rule, as a sum of Schur functions $s_{\alpha}$ for partitions $\alpha$ such that $\alpha_{1} \leq l[13$, p. 69].

Similarly, we have $\psi_{p}\left(s_{\mu / \nu}\right)=\varepsilon(\mu) \varepsilon(\nu) s_{\mu(r) / \nu(r)} \prod_{k \neq r} s_{\mu(k)}$. If for some $k \neq r$, $\mu(k)_{1}>l$, then on expanding this product as a sum of Schur functions by the Littlewood-Richardson rule, every $s_{\beta}$ which appears corresponds to a partition $\beta$ with $\beta_{1}>l$.

By orthogonality of Schur functions, it follows that $\left\langle\psi_{p}\left(s_{\lambda^{\prime} / \nu^{\prime}}\right), \psi_{p}\left(s_{\mu / \nu}\right)\right\rangle=$ 0 . Since this is true for all $\nu$, the result now follows from Proposition 2.7.

In certain cases, it is possible to obtain a relation between the transition matrices $M\left(s^{\prime}, s\right)$ themselves for blocks with different cores. Our next result was suggested by the work of Scopes [17], who gives a sufficient condition for blocks of $p$-modular representations of the same weight in the symmetric groups $S_{d}$ and $S_{d^{\prime}}$ to be Morita equivalent. Although this theorem makes sense only when $p$ is prime, the condition found by Scopes is purely combinatorial, and makes sense for any modulus $p$.

Thus, for any $p \geq 2$, let $\nu$ be a core of degree $d$, and suppose that, in the abacus representation of $\nu$, there are $W$ more beads on some runner, say the $k$ th, than on the one immediately below, the $(k-1)$ th. (Recall that the runners correspond to residue classes mod $p$. Here the numbering is interpreted cyclically, i.e. the position numbered $k$ is on the $k$ th runner, for all $k \geq 0$.) Let $\bar{\nu}$ denote the core of degree $d-W$ which is obtained by exchanging the beads on these two runners. Then for all $w \leq W$, when $p$ is prime, the block $B$ of $S_{d+p w}$ with core $\nu$ and the block $\bar{B}$ of $S_{d-W+p w}$ with core $\bar{\nu}$ are Morita equivalent [17, Theorem 4.2].

For example, the 3-blocks of $S_{12}$ with core $(4,2)$, of $S_{10}$ with core $(3,1)$, and of $S_{8}$ with core (2) are all Morita equivalent.

The Morita equivalence is constructed by means of an explicit bijection $S: B \rightarrow \bar{B}$ between the partitions in the two blocks. In the abacus representation, $S$ interchanges the beads on the $i$ th and $(i-1)$ th runners. We shall refer to $S$ as a Scopes equivalence of weight $W$. In [17, Lemma 2.2], it is proved that $S$ is in fact the bijection which preserves the lexicographic order of the partitions.

Proposition 2.12. Let $p \geq 2$ and let $S: B \rightarrow \bar{B}$ be a Scopes equivalence. Then for all $\lambda, \mu \in B,\left\langle s_{\lambda}^{\prime}, s_{\mu}\right\rangle=\left\langle s_{S(\lambda)}^{\prime}, s_{S(\mu)}\right\rangle$; i.e. when the partitions in both blocks are ordered lexicographically, the transition matrices $M\left(s^{\prime}, s\right)$ for the blocks $B$ and $\bar{B}$ are identical.

Proof. Clearly, the effect of $S$ on quotients is to exchange the $i$ th and $(i-1)$ th components. We shall prove that $S$ preserves the sign. To see this, recall [8, $\S 2.7]$ that $\varepsilon(\lambda)=(-1)^{s}$, where $s$ is the sum over all beads of the number of beads passed by each bead in turn as it is moved up the abacus to its position in the abacus representation of the core $\nu$. 
Interchanging the $i$ th and $(i-1)$ th runners does not affect this count so far as beads on any other runner are concerned. Thus it suffices to consider the case where there are only two runners. Let $W$ be the weight of the equivalence $S$, and let $w$ be the weight of the blocks $B$ and $\bar{B}$, so that $w \leq W$. Because there are $W$ more beads on one runner than the other, and no more than $w$ moves of beads up the abacus from the core are allowed, no bead on the 'shorter' runner can reach a row higher than that containing the lowest bead on the 'longer' runner that is not moved. Thus moves on the 'longer' runner make no contribution to $s$, while moves through $k$ places on the 'shorter' runner make the maximum contribution $k$ to $s$, irrespective of the relative positions of the two runners. Thus the sign is the same for two partitions which correspond under $S$.

It now follows from Proposition 2.3 that if $\lambda$ and $\mu$ are partitions with the same core $\nu$ and weights $w(\mu) \leq w(\lambda) \leq W$, and if $S$ is a Scopes equivalence of weight $W$ from blocks with core $\nu$ to blocks with core $\bar{\nu}=S(\nu)$, then $\psi_{p}\left(s_{\lambda / \mu}\right)=\psi_{p}\left(s_{S(\lambda) / S(\mu)}\right)$. Next, we observe that the terms in the summation in Proposition 2.7 correspond under $S$. Thus we can apply this result to the sum term by term, proving that $\left\langle s_{\lambda}^{\prime}, s_{\mu}\right\rangle=\left\langle s_{S(\lambda)}^{\prime}, s_{S(\mu)}\right\rangle$.

For the statement about lexicographic order, we rely on Scopes's argument, noting that this does not depend on $p$ being prime. This completes the proof.

We conclude this section by considering contragredient duality for Schur functions and modular Schur functions. This provides an additional tool for attacking Conjecture 1.4, and we illustrate this by giving a proof of this conjecture for the case $n=3$.

We recall some basic facts from e.g. [5]. The irreducible polynomial representations of $G L(n, K)$, where $K$ is an infinite field, are indexed by partitions $\lambda$ of length $\leq n$, which are their highest weights. The ring of $\mathbf{Z}$-linear combinations of these representations is therefore naturally identified with $\Lambda_{n}$. If $K$ has characteristic 0 , then the formal character of the irreducible representation with highest weight $\lambda$ is the Schur function $s_{\lambda}$. The determinant representation corresponds to the $n$th elementary symmetric function $e_{n}$.

We can also consider the irreducible rational representations of $G L(n, K)$. These can all be obtained as the tensor product of a polynomial representation with an integer power of the determinant. Hence the corresponding rational representation ring is $\Lambda_{n}^{*}=\mathrm{Z}\left[e_{1}, e_{2}, \ldots, e_{n}, e_{n}^{-1}\right]$, and is isomorphic to the tensor product of the polynomial ring $\Lambda_{n-1}$ with the ring of Laurent polynomials in $e_{n}$.

Contragredient duality (defined using $g \mapsto g^{-1}$ ) defines an involution on $\Lambda_{n}^{*}$, which is in fact the involution $\psi^{-1}$ given by $x_{i} \mapsto x_{i}^{-1}, 1 \leq i \leq n$; cf. [1, $\S 6]$.

The Z-basis $\left\{m_{\lambda} \mid l(\lambda) \leq n\right\}$ of monomial symmetric functions for $\Lambda_{n}$ extends to a $\mathbf{Z}$-basis $\left\{e_{n}^{k} m_{\lambda} \mid l(\lambda) \leq n-1, k \in \mathbf{Z}\right\}$ for $\Lambda_{n}^{*}$. For $k \geq 0$, we have $e_{n}^{k} m_{\lambda}=m_{\lambda+\left(k^{n}\right)}$, and we extend this notation by allowing $k$ to be a negative integer.

Thus, for any monotonic decreasing $n$-term sequence of integers $\alpha=\left(\alpha_{1}, \alpha_{2}\right.$, $\left.\ldots, \alpha_{n}\right)$, we extend the usual notation for partitions $[13$, p. 11] by writing

$$
m_{\alpha}=\sum x_{1}^{\alpha_{1}} x_{2}^{\alpha_{2}} \cdots x_{n}^{\alpha_{n}},
$$


where the sum is over all distinct permutations of the terms of $\alpha$. Then $\alpha$ can be uniquely written as $\lambda+\left(k^{n}\right)$, where $\lambda$ is a partition of length $\leq n-$ 1 and $k \in \mathbf{Z}$, so that $m_{\alpha}=e_{n}^{k} m_{\lambda}$. Thus $\left\{m_{\alpha}\right\}$ is a $\mathbf{Z}$-basis for $\Lambda_{n}^{*}$. In terms of this basis, the action of $\psi^{-1}$ is given by $\psi^{-1} m_{\alpha}=m_{\bar{\alpha}}$, where $\bar{\alpha}=$ $\left(-\alpha_{n},-\alpha_{n-1}, \ldots,-\alpha_{1}\right)$.

The Z-basis $\left\{s_{\lambda} \mid l(\lambda) \leq n\right\}$ of Schur functions for $\Lambda_{n}$ similarly extends to a Z Z-basis $\left\{e_{n}^{k} s_{\lambda} \mid l(\lambda) \leq n-1, k \in \mathbf{Z}\right\}$ for $\Lambda_{n}^{*}$. It is clear from the fact that multiplication by $e_{n}$ corresponds to taking the tensor product of a representation with the determinant representation that, for $k \geq 0, e_{n}^{k} s_{\lambda}=s_{\lambda+\left(k^{n}\right)}$. This can also be seen by an easy combinatorial argument using the formula $s_{\lambda}=\sum_{\lambda \triangleright \mu} K_{\lambda, \mu} m_{\mu}$, since $m_{\mu} e_{n}^{k}=m_{\mu+\left(k^{n}\right)}$ and $K_{\lambda, \mu}=K_{\lambda+\left(k^{n}\right), \mu+\left(k^{n}\right)}$. This relation between Kostka numbers is seen by observing that there is a unique way to add $k$ columns of length $n$ to each tableau of shape $\lambda$ and content $\mu$.

We extend the notation for Schur functions and Kostka numbers in the same way as for the monomial symmetric functions. Thus, for any monotonic decreasing $n$-term sequence of integers $\alpha=\left(\alpha_{1}, \alpha_{2}, \ldots, \alpha_{n}\right)$, let $\alpha=\lambda+\left(k^{n}\right)$, where $\lambda$ is a partition of length $\leq n-1$ and $k \in \mathbf{Z}$, and let $s_{\alpha}=e_{n}^{k} s_{\lambda}$. Then $\left\{s_{\alpha}\right\}$ is a $\mathbf{Z}$-basis for $\Lambda_{n}^{*}$.

Notice that the relation $s_{\alpha}=e_{n}^{k} s_{\lambda}$ holds for any partition $\lambda$ and integer $k$ such that $\alpha=\lambda+\left(k^{n}\right)$ and $l(\lambda) \leq n$. Thus if $\beta$ is also an $n$-term monotonic decreasing sequence of integers, where $\beta=\mu+\left(k^{n}\right)$ and $l(\mu) \leq n$, we may define the Kostka number $K_{\alpha, \beta}=K_{\lambda, \mu}$, since, as observed above, this number does not depend on the choice of $k$. We also extend the dominance partial ordering in the obvious way, so that $\alpha \unrhd \beta$ if and only if $\lambda \unrhd \mu$.

It follows immediately from these definitions that we have

$$
s_{\alpha}=\sum_{\alpha \unrhd \beta} K_{\alpha, \beta} s_{\beta} .
$$

We use this formula to calculate the action of $\psi^{-1}$ on $\Lambda_{n}^{*}$ in terms of the basis of Schur functions.

Lemma 2.13. For any monotonic decreasing sequence of integers $\alpha=\left(\alpha_{1}, \alpha_{2}\right.$, $\left.\ldots, \alpha_{n}\right)$,

$$
\psi^{-1} s_{\alpha}=s_{\bar{\alpha}} .
$$

Proof. We shall prove that $K_{\alpha, \beta}=K_{\bar{\alpha}, \bar{\beta}}$. The result follows from this using the formula above, since $\psi^{-1} m_{\beta}=s_{\bar{\beta}}$ for each sequence $\beta$. Note that $\alpha \unrhd \beta$ if and only if $\bar{\alpha} \unrhd \bar{\beta}$.

Choose partitions $\lambda$ and $\mu$ of length $\leq n$ and $k \in \mathbf{Z}$ such that $\alpha=\lambda+\left(k^{n}\right)$ and $\beta=\mu+\left(k^{n}\right)$, and similarly choose partitions $\lambda^{*}$ and $\mu^{*}$ of length $\leq n$ and $l \in \mathbf{Z}$ such that $\bar{\alpha}=\lambda^{*}+\left(l^{n}\right)$ and $\bar{\beta}=\mu^{*}+\left(l^{n}\right)$. Then $K_{\alpha, \beta}=K_{\lambda, \mu}$ and $K_{\bar{\alpha}, \bar{\beta}}=K_{\lambda^{*}, \mu^{*}}$. If $i+j=n+1$, then $\lambda_{i}+\lambda_{j}^{*}=\left(\alpha_{i}-k\right)+\left(-\alpha_{i}-l\right)=N$, where $N=-k-l \geq 0$ since $\lambda$ and $\lambda^{*}$ are partitions. In terms of Young diagrams, this relation means that the diagram $\lambda^{*}$ is obtained from the diagram $\lambda$ by turning its complement in the rectangle $\left(N^{n}\right)$ upside down. Similarly, $\mu_{i}+\mu_{j}^{*}=N$, so the diagrams of $\mu^{*}$ and $\mu$ are related in the same way.

Given a semistandard tableau of shape $\lambda$ and content $\mu$, there is a unique way to extrend it to a tableau of shape $\left(N^{n}\right)$ in which each column is a permutation of $1,2, \ldots, n$ and the 'complementary tableau' of shape $\lambda^{*}$ is semi- 
standard. However, the content of this tableau is not $\mu^{*}$, but its reversal $\left(N^{n}\right)-\mu$. We illustrate this construction by an example.

Example 2.14. Let $n=4$ and let $\lambda=(5,4,2,0)$ so that $\bar{\lambda}=(0,-2,-4,-5)$. The upper part of the tableau below is a semistandard tableau of shape $\lambda$ and content $\mu=\left(3^{3}, 2\right)$. The lower part is a semistandard tableau of shape $\lambda^{*}=(5,3,1,0)=\bar{\lambda}+\left(5^{4}\right)$ and content $\left(5^{4}\right)-\mu=\left(2^{3}, 3\right)$ (turned upside down).

\begin{tabular}{|lllll|}
\hline 1 & 1 & 1 & 2 & 3 \\
\cline { 5 - 5 } 2 & 2 & 3 & 4 & 4 \\
\cline { 4 - 5 } 3 & 4 & 4 & 3 & 2 \\
\cline { 5 - 6 } 4 & 3 & 2 & 1 & 1 \\
\hline
\end{tabular}

This construction defines a bijection between the set of semistandard tableaux of shape $\lambda$ and content $\mu$ and the set of semistandard tableaux of shape $\lambda^{*}$ and content $\left(N^{n}\right)-\mu$. However, permuting the terms of the content does not change the number of semistandard tableaux, by $[13, \mathrm{I}(5.12)]$, and the partition $\mu^{*}$ is the reverse of the sequence $\left(N^{n}\right)-\mu$. Hence $K_{\lambda, \mu}=K_{\lambda^{*}, \mu^{*}}$.

We next consider the action of $\psi^{-1}$ on the modular Schur function $s_{\lambda}^{\prime}$, where $\lambda$ is a partition of length $\leq n$. Let $N=n(p-1)$. Since $s_{\lambda}^{\prime}=0$ if $\lambda_{1}>N$, we shall assume that $\lambda_{1} \leq N$. As above, we define the 'complementary' partition $\lambda^{*}$ by $\lambda_{i}+\lambda_{j}^{*}=N$ if $i+j=n+1$.

Lemma 2.15. For any partition $\lambda$ with $l(\lambda) \leq n$ and $\lambda_{1} \leq n(p-1)$,

$$
\psi^{-1} s_{\lambda}^{\prime}=e_{n}^{-n(p-1)} s_{\lambda}^{\prime} .
$$

Proof. Since $h_{d}^{\prime}$ is the sum of all monomial symmetric functions with all exponents $<p$, it is easy to see that $h_{N}^{\prime}=e_{n}^{p-1}$ and that $h_{N-d}^{\prime}=h_{N}^{\prime} \psi^{-1}\left(h_{d}^{\prime}\right)$ for $0 \leq d \leq N$. This is the case $\lambda=(d)$ of the lemma. For the general case, we have

$$
\begin{aligned}
\psi^{-1} s_{\lambda}^{\prime} & =\psi^{-1} \operatorname{det}\left(h_{\lambda_{i}-i+j}^{\prime}\right)=\operatorname{det}\left(\psi^{-1}\left(h_{\lambda_{i}-i+j}^{\prime}\right)\right) \\
& =\left(h_{N}^{\prime}\right)^{-n} \operatorname{det}\left(h_{N-\lambda_{i}+i-j}^{\prime}\right)=\left(h_{N}^{\prime}\right)^{-n} \operatorname{det}\left(h_{\lambda_{i}^{*}-i+j}^{\prime}\right)
\end{aligned}
$$

by reversing the order of the rows and columns, and this determinant is $s_{\lambda^{*}}^{\prime}$.

From Lemma 2.13 we have a similar formula $\psi^{-1} s_{\lambda}=e_{n}^{-n(p-1)} s_{\lambda^{*}}$. We can exploit the similarity of these formulae to obtain a further symmetry of the matrices $M\left(s^{\prime}, s\right)$.

Proposition 2.16. Let $\lambda$ and $\mu$ be partitions of length $\leq n$ and with all parts $\leq n(p-1)$. Then

$$
\left\langle s_{\lambda}^{\prime}, s_{\mu}\right\rangle=\left\langle s_{\lambda^{*}}^{\prime}, s_{\mu^{*}}\right\rangle .
$$

Proof. Applying $\psi^{-1}$ to the formula $s_{\lambda}^{\prime}=\sum_{\mu} m_{\lambda, \mu} s_{\mu}$, we have

$$
e_{n}^{-n(p-1)} s_{\lambda^{*}}^{\prime}=\sum_{\mu} m_{\lambda, \mu} e_{n}^{-n(p-1)} s_{\mu^{*}},
$$


and cancelling the unit $e_{n}^{-n(p-1)}$ this gives $m_{\lambda, \mu}=m_{\lambda^{*}, \mu^{*}}$, from which the result follows by orthonormality of Schur functions.

This result reduces the work in checking Conjecture 1.4 for the $n$ variable case by half. For this purpose, we consider the abacus representation of partitions of length $\leq n$ and all parts $\leq n(p-1)$ by using $b=n$ beads on the first $n$ rows of the abacus. (Thus the total number of such partitions is $\left(\begin{array}{c}n p \\ n\end{array}\right)$; cf. [4, Proposition 2.7]. The abacus representation of $\lambda^{*}$ is then obtained from that of $\lambda$ simply by turning the abacus upside down. Hence $\lambda^{*}$ and $\mu^{*}$ are in the same block if and only if $\lambda$ and $\mu$ are in the same block.

The maximum weight of a partition that can be represented in this way is $n(n-1)$. It is easy to see that if there are $c_{k}$ beads on the $k$ th runner, then $w(\lambda)+w\left(\lambda^{*}\right)=\sum_{k=0}^{p-1} c_{k}\left(n-c_{k}\right) \leq n(n-1)$. To prove Conjecture 1.4 for a given value of $n$, it is therefore sufficient to consider partitions such that $w(\lambda) \leq w\left(\lambda^{*}\right)$. In particular, we need only consider blocks of weight $\leq n(n-1) / 2$.

For example, in the case $n=2$ it suffices to consider cores and blocks of weight 1 . The results of Section 1 therefore imply that Conjecture 1.4 is true in this case. (Another proof is given in $[4, \S 3.9]$.)

Proposition 2.17. If Conjecture 1.4 is true for $\Lambda_{n}$ for $2 \leq p \leq n$, then it is true for $\Lambda_{n}$ for all $p \geq 2$. In particular, Conjecture 1.4 is true for $n=3$.

Proof. We have a $n \times p$ abacus, i.e. $p$ runners with $n$ positions on each, numbered as in Section 0 , and we consider only those partitions which are represented in this way. Hence runners carrying no beads do not affect the calculation of the $A$ matrix of Corollary 2.10 for a particular block by means of Proposition 2.9. Thus every $A$ matrix which occurs for a block for any $p$ already occurs for a block when $p$ is the number of nonempty runners. This proves the first statement.

Now let $n=3$. Since Conjecture 1.4 is already proved for $p=2$, we may assume that $p=3$ and that there is one bead on each of the three runners. Hence we may assume that the core is $(0)$. The results of Section 1 deal with blocks of weight 0 or 1 , so using Proposition 2.16 we are reduced to considering the blocks of weights 2 and 3 with core $(0)$ for $p=3$.

The $A$ matrices for these blocks (with $n=3$ ) are easily calculated from Proposition 2.9, and are shown below (Tables 1 and 2). For each pair $\lambda, \mu$ the value of $a_{\lambda, \mu}$ is tabulated, $\lambda$ being given in quotient notation and $\mu$ in partition notation. (Parentheses indicating partitions are omitted; for example $(0,0,2)$ means quotient $((0),(0),(2))$, i.e. the $\beta$-numbers $8,1,0$ for the partition (6).)

The determinants are -1 and 4 respectively, so they are nonsingular.

Remark 2.18. The values of the determinant of the matrix $K^{\prime}$ given in [4, p. 11] can be verified by the methods we have developed. For example, for $n=3$ the only blocks whose $A$ matrix has determinant other than 1 or -1 are the blocks of weight 1,3 or 5 with beads on 3 runners, for which the determinant is $-2,4,-2$ respectively. There are $\left(\begin{array}{l}p \\ 3\end{array}\right)$ blocks of each type, so $\operatorname{det} K^{\prime}=\operatorname{det} M\left(s^{\prime}, s\right)= \pm 16\left(\begin{array}{c}p \\ 3\end{array}\right)$. 
TABLE 1

\begin{tabular}{|c|cccccc|}
\hline & 6 & 51 & $41^{2}$ & $3^{2}$ & 321 & $2^{3}$ \\
\hline$(0,0,2)$ & 0 & 0 & 0 & 0 & 0 & 1 \\
$(0,2,0)$ & 0 & 0 & 0 & 0 & 1 & 0 \\
$(2,0,0)$ & 0 & 0 & 0 & 1 & 0 & 0 \\
$(0,1,1)$ & 0 & 0 & 1 & 1 & 1 & 1 \\
$(1,0,1)$ & 0 & 1 & 0 & 1 & 1 & 1 \\
$(1,1,0)$ & 1 & 0 & 0 & 1 & 1 & 1 \\
\hline
\end{tabular}

TABLE 2

\begin{tabular}{|c|ccccccc|}
\hline & 63 & 54 & 621 & $52^{2}$ & $4^{2} 1$ & 432 & $3^{3}$ \\
\hline$(0,1,2)$ & 0 & 0 & 0 & 0 & 0 & 1 & 1 \\
$(0,2,1)$ & 0 & 0 & 0 & 0 & 1 & 0 & 1 \\
$(1,0,2)$ & 0 & 0 & 0 & 1 & 0 & 0 & 1 \\
$(1,2,0)$ & 0 & 0 & 1 & 0 & 0 & 0 & 1 \\
$(2,0,1)$ & 0 & 1 & 0 & 0 & 0 & 0 & 1 \\
$(2,1,0)$ & 1 & 0 & 0 & 0 & 0 & 0 & 1 \\
$(1,1,1)$ & 1 & 1 & 1 & 1 & 1 & 1 & 2 \\
\hline
\end{tabular}

\section{Highest WEIGHTS}

In this section we shall show that certain entries $\left\langle s_{\lambda}^{\prime}, s_{\mu}\right\rangle$ in the transition matrices $M\left(s^{\prime}, s\right)$ are zero. We shall consider two special types of partition, which we call row stable and horizontal partitions.

By the highest weight of a homogeneous symmetric function $f \in \Lambda$ of degree $d$, we mean the maximal partition $\rho$ of $d$ in the lexicographic ordering such that the coefficient of the monomial $x_{1}^{\rho_{1}} x_{2}^{\rho_{2}} \cdots x_{l}^{\rho_{l}}$ in $f$ is nonzero, where $l=$ $l(\rho)$. Equivalently, if $f=\sum_{\sigma} c_{\sigma} m_{\sigma}$ in terms of the Z-basis of monomial symmetric functions, then $c_{\rho} \neq 0$, and if $c_{\sigma} \neq 0$ then $\sigma \leq \rho$. Since the highest weight of the product $m_{\rho} m_{\sigma}$ is $\rho+\sigma$, it follows that the highest weight of any product of symmetric functions is the sum of their highest weights.

The Kostka matrix $K=M(s, m)$ is 'strictly upper unitriangular' [13, I (6.5)], i.e. the Schur function $s_{\mu}=m_{\mu}+\sum_{\sigma \triangleleft \mu} K_{\mu, \sigma} m_{\sigma}$ has highest weight $\mu$, and all other 'weights' $\sigma$ are dominated by $\mu$. Since the Schur functions form an orthonormal basis for $\Lambda$, the highest weight $\rho$ of any symmetric function $f \in \Lambda$ is also the maximal partition in the lexicographic order with the property that $\left\langle f, s_{\rho}\right\rangle \neq 0$.

All of this also applies to a homogeneous symmetric polynomial $f \in \Lambda_{n}$, using the Z-basis $\left\{m_{\lambda} \mid l(\lambda) \leq n\right\}$ and the orthonormal basis $\left\{s_{\lambda} \mid l(\lambda) \leq n\right\}$.

For example, the highest weight of $h_{d}^{\prime}$ is $\tau(d)=(p-1, \ldots, p-1, b)$, with $a$ parts equal to $p-1$, where $d=(p-1) a+b$ and $0 \leq b<p-1$. Hence the highest weight of $h_{\lambda}^{\prime}$ is $\tau(\lambda)=\sum_{i} \tau\left(\lambda_{i}\right)$. The diagram of $\tau(\lambda)$ is shown schematically in Figure 1. (The actual Young diagram may not look like this, because nodes may have to be moved to the left to fill gaps when adding the partitions $\tau\left(\lambda_{i}\right)$.) If $p=2$, then of course $\tau(\lambda)=\lambda^{\prime}$.

It is easy to see that if $\lambda \unlhd \mu$ then $\tau(\lambda) \unrhd \tau(\mu)$. For this, we need only consider the effect of raising the last node in the $j$ th row in $\lambda$ to the end of the 


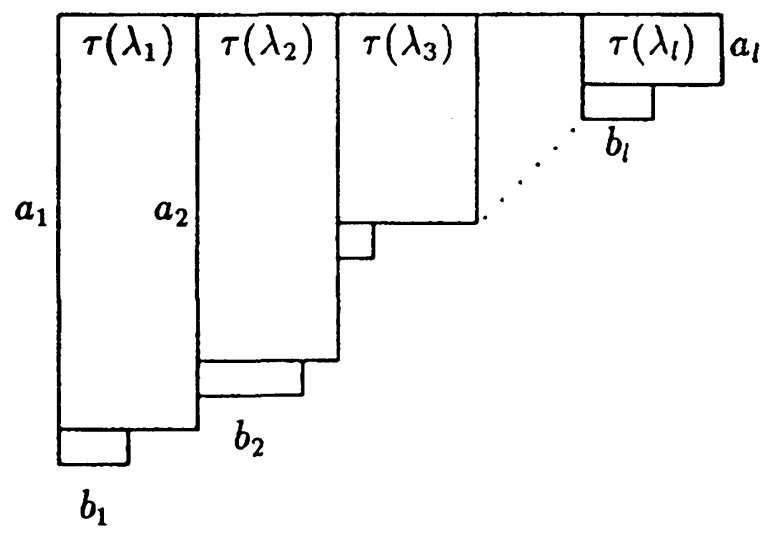

FIGURE 1

$i$ th row, where $i \leq j$. The node added to $\tau\left(\lambda_{i}\right)$ cannot lie in a higher row than the node removed from $\tau\left(\lambda_{j}\right)$, although it may lie in the same row. Summing over $i$, the effect on $\tau(\lambda)$ is to lower it in the dominance partial order or leave it unchanged.

We apply this observation to obtain an upper bound on the highest weight of an arbitrary modular Schur function.

Proposition 3.1. For any partition $\lambda$, if $\rho$ is the highest weight of $s_{\lambda}^{\prime}$, then $\rho \leq \tau(\lambda)$.

Proof. We can regard the Jacobi-Trudi expansion of $s_{\lambda}^{\prime}$ as an alternating sum $\left[13, \mathrm{I}\left(3.4^{\prime}\right)\right]$

$$
s_{\lambda}^{\prime}=\sum_{\pi \in S_{l}}(-1)^{\operatorname{sgn}(\pi)} h_{\lambda+\delta-\pi(\delta)}^{\prime},
$$

where $\delta$ is the sequence $(l-1, l-2, \ldots, 1,0)$, and $\pi$ permutes the terms of $\delta$. We shall prove that the highest weight of each term in this sum is $\leq \tau(\lambda)$.

Since $h_{d}^{\prime}=0$ if $d<0$, we may assume that the terms of the sequence $\lambda+\delta-$ $\pi(\delta)$ are $\geq 0$. Let $\pi(\lambda)$ be the partition obtained by arranging this sequence in decreasing order, so that $h_{\lambda+\delta-\pi(\delta)}^{\prime}=h_{\pi(\lambda)}^{\prime}$ has highest weight $\tau(\pi(\lambda))$. By [13, I (1.13)], $\pi(\lambda) \unrhd \lambda$, and, as remarked above, it follows that $\tau(\pi(\lambda)) \unlhd \tau(\lambda)$, and hence $\tau(\pi(\lambda)) \leq \tau(\lambda)$.

We can refine this argument so as to show that this bound is exact in certain cases. Recall (Section 0 ) that we define the partition $\lambda$ to be row stable if its parts differ by at least $p-1$, i.e. if $\lambda_{i}-\lambda_{i+1} \geq p-1$ for $1 \leq i \leq l-1$, where $l=l(\lambda)$. (Note that the last part $\lambda_{l}$ is not required to be $\geq p-1$.)

Proposition 3.2. If at most one part of $\lambda$ lies in any open interval of the form $(a(p-1),(a+1)(p-1))$, then the highest weight of $s_{\lambda}^{\prime}$ is $\tau=\tau(\lambda)$, with coefficient $\left\langle s_{\lambda}^{\prime}, s_{\tau}\right\rangle=1$. In this case, $\tau$ is column regular if and only if $\lambda$ is row stable.

Proof. We modify the proof of Proposition 3.1. Let $\lambda$ satisfy the additional hypothesis, and suppose that $\pi$ is not the identity permutation. Then, since $\delta$ has distinct parts, $\pi(\delta) \triangleleft \delta$, and hence $\pi(\lambda) \triangleright \lambda$. Consider again the effect 


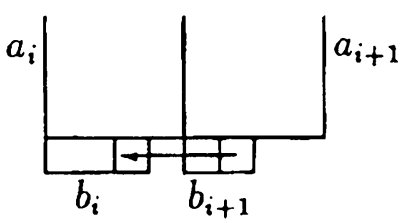

FIGURE 2

of raising the last node in the $j$ th row in $\lambda$ to the end of the $i$ th row, where $i \leq j$. This adds a node to $\tau\left(\lambda_{i}\right)$ and removes one from $\tau\left(\lambda_{j}\right)$. Now the condition on $\lambda$ ensures that the node added cannot lie in the same row as the node removed. To see this, it is sufficient to consider two adjacent rows of $\lambda$. For any $j$, let $\lambda_{j}=(p-1) a_{j}+b_{j}$, where $0 \leq b_{j}<p-1$. Figure 2 illustrates the situation we wish to rule out.

This diagram shows the situation where $a_{i}=a_{i+i}=a$ and $0<b_{i+1} \leq b_{i}<$ $p-1$, i.e. $a(p-1)<\lambda_{i+1} \leq \lambda_{i}<(a+1)(p-1)$, and this is contrary to our hypothesis.

It follows that $\tau(\pi(\lambda)) \triangleleft \tau(\lambda)$. Hence the leading term $h_{\lambda}^{\prime}$ in the Jacobi-Trudi expansion of $s_{\lambda}^{\prime}$ has highest weight $\tau=\tau(\lambda)$, with coefficient 1 , and all other terms in the expansion have a highest weight which is $\triangleleft \tau$ and hence $<\tau$. Hence $\tau$ is the highest weight of $s_{\lambda}^{\prime}$, with coefficient $\left\langle s_{\lambda}^{\prime}, s_{\tau}\right\rangle=1$.

Finally, we check the statements about column regularity of $\tau$. With $\lambda$ as stated, if $\lambda_{i}-\lambda_{i+1}<p-1$ then, for some $a$, either $a(p-1)<\lambda_{i}<(a+1)(p-1)$ and $(a-1)(p-1)<\lambda_{i+1}<a(p-1)$ or at least one of $\lambda_{i}$ and $\lambda_{i+1}$ is $a(p-1)$. In all these cases it is easy to see that $\tau$ is column singular. In the remaining cases, $\lambda$ is row stable. Then $a_{i}>a_{i+1}$, and $a_{i}=a_{i+1}+1$ if and only if $b_{i} \geq b_{i+1}$. Again, it is easy to see that these conditions imply that $\tau$ is column regular.

Remark 3.3. By Corollary 2.8, the highest weight of $s_{\lambda}^{\prime}$ must be a partition in the same block as $\lambda$. The partition $\tau(\lambda)$ is not in general in the same block, and so the bound given by Proposition 3.1 cannot be exact. For example, let $p=3, \lambda=(5,1,1)$; then $\tau=(4,2,1)$, but the highest weight of $s_{\lambda}^{\prime}$ is $\left(3^{2}, 1\right)$.

For certain special row stable partitions $\lambda$ whose core is a Steinberg partition, we can say more. Recall (Section 0 ) that we call a partition $\tau$ strongiy column regular if $\tau<\mu$ for all column singular $\mu$ in the same block. If the highest weight of a homogeneous symmetric function $f$ of degree $d$ is strongly column regular, then $f$ is a Z-linear combination of monomial symmetric functions $m_{\sigma}$, where each $\sigma$ is a strongly column regular partition of $d$. Equivalently, if $\left\langle s_{\lambda}^{\prime}, s_{\sigma}\right\rangle \neq 0$, then $\sigma$ is strongly column regular.

Proposition 3.4. Let $\lambda=\nu+p \alpha$, where $\alpha$ is a partition of length $\leq l$ and $\nu$ is the Steinberg partition $((l-1)(p-1),(l-2)(p-1), \ldots, p-1)$. Then $\tau=\tau(\lambda)$ is the highest weight of $s_{\lambda}^{\prime}$, and $\tau$ is strongly column regular. If $\sigma$ is a partition with core $\nu$ and weight $|\alpha|$ and $\sigma \leq \tau$, then, in the abacus representation using $b$ beads where $b \equiv l \bmod p$, the 0 th component of the quotient of $\sigma$ is 0 .

Proof. First take $b=l$. Then, for $1 \leq i \leq l$, the $i$ th $\beta$-number of $\lambda$ is $(l-i)(p-1)+p \alpha_{i}+(l-i)^{\circ}=\left(l-i+\alpha_{i}\right) p$, and hence the quotient of $\lambda$ is $(\alpha,(0), \ldots,(0))$. 
Since $\lambda_{i}=(l-i)(p-1)+p \alpha_{i}, \lambda_{i}-\lambda_{i+1}=p-1+p\left(\alpha_{i}-\alpha_{i+1}\right) \geq p-1$, so $\lambda$ is row stable. By Proposition 3.2, $\tau$ is column regular and is the highest weight of $s_{\lambda}^{\prime}$. As in the proof of Proposition 3.2, we write $\lambda_{i}=(p-1) a_{i}+b_{i}$, where $0 \leq b_{i}<p-1$.

Consider the abacus representation of $\tau$. Part of the statement to be proved is that the 0 th component of the quotient of $\tau$ is $(0)$. This is equivalent to the statement that there are no gaps in the sequence of $\beta$-numbers of $\tau$ on the 0 th runner. Since $\tau$ is column regular, there are no gaps of length $\geq p$ between consecutive $\beta$-numbers, so this is equivalent to showing that every integer $\equiv 0$ $\bmod p$ and less than some $\beta$-number of $\tau$ is itself a $\beta$-number of $\tau$.

We first set up the number of beads $b$ that we require on the abacus. This must satisfy $b \geq l(\tau)$ and $b \equiv l \bmod p$. Since $a_{1}(p-1)+b_{1}=\lambda_{1}=p \alpha_{1}+$ $(l-1)(p-1)$, we have $a_{1}-b_{1}+1 \equiv l \bmod p$, so that we may choose $b=$ $\left(a_{1}+1\right)+\left(p-b_{1}\right)$.

Now consider the $\beta$-number for the last part of $\tau$. This part is $b_{1}$ if $b_{1}>0$, and the number of 'extra' beads used is $b-l(\tau)=b-\left(a_{1}+1\right)=p-b_{1}$, so this $\beta$ number is $b_{1}+\left(p-b_{1}\right)=p$, and the lower $\beta$-numbers are $p-b_{1}-1, \ldots, 1,0$. In the case where $b_{1}=0$, the last part is $p-1$, and the number of 'extra' beads is $b-l(\tau)=b-a_{1}=p+1$, so this $\beta$-number is $(p-1)+(p+1)=2 p$, and the lower $\beta$-numbers are $p, p-1, \ldots, 1,0$. Note that, in both cases, the $\left(a_{1}+1\right)$ th $\beta$-number is $p$.

Next consider the $\beta$-number for the $\left(a_{i}+1\right)$ th row of $\tau$, where $1 \leq i \leq l$. Let the hook length of the first node $x_{i}$ in this row be $c_{i}$. Then it is clear from Figure 3 that $c_{i+1}-c_{i}=\left(a_{i}-a_{i+1}\right)+(p-1)+\left(b_{i+1}-b_{i}\right)$.

Since $\lambda_{i}=(p-1) a_{i}+b_{i}$, and $\lambda_{i}-\lambda_{i+1} \equiv-1 \bmod p$, it follows that $c_{i+1} \equiv c_{i}$ $\bmod p$. Hence the $\left(a_{i}+1\right)$ th and $\left(a_{i+1}+1\right)$ th $\beta$-numbers of $\tau$ are congruent $\bmod p$. Since the $\left(a_{1}+1\right)$ th $\beta$-number is $p$, the $\left(a_{i}+1\right)$ th $\beta$-number is $\equiv 0$ $\bmod p$, for $1 \leq i \leq l$.

We next show that any multiple of $p$ which lies between the $\left(a_{i}+1\right)$ th and $\left(a_{i+1}+1\right)$ th $\beta$-numbers must also be a $\beta$-number of $\tau$. If $a_{i}=a_{i+1}+1$, then $b_{i}=b_{i+1}$, since $\lambda_{i}-\lambda_{i+1} \equiv-1 \bmod p$, so $\tau_{a_{i}}-\tau_{a_{i}+1}=p-1$ and the two $\beta$-numbers differ by $p$. Otherwise, as we pass from the $\left(a_{i}+1\right)$ th to the $\left(a_{i+1}+1\right)$ th $\beta$-number, consecutive $\beta$-numbers increase by $p-b_{i}, 1,1, \ldots, 1$, $b_{i+1}+1$, and again no multiple of $p$ is passed over; and similarly for the $\beta$ numbers greater than the $\left(a_{l}+1\right)$ th .

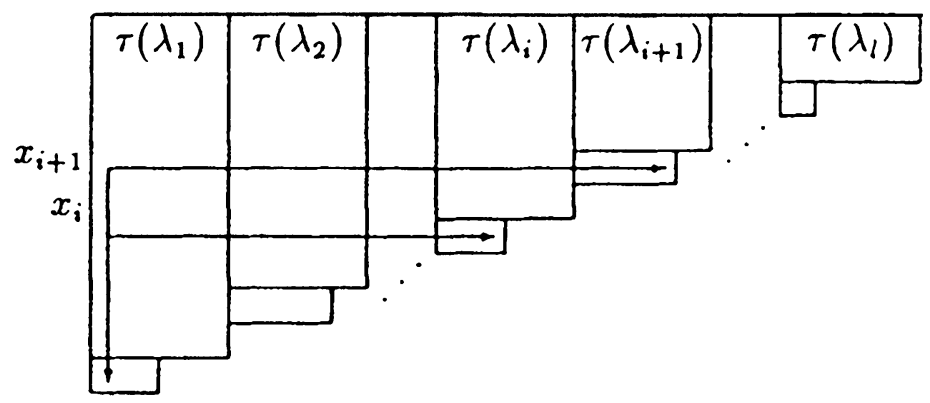

FIGURE 3 
Hence there are no gaps in the sequence of $\beta$-numbers of $\tau$ on the 0 th runner, and so the 0th component of the quotient of $\tau$ is 0 .

Let $\sigma$ be a partition in the same block as $\tau$, with $\sigma \leq \tau$. Using $b$ beads, where we assume $b \geq l(\sigma)$ as well as $b \geq l(\tau)$ and $b \equiv l \bmod p$, the abacus representation for $\sigma$ can be obtained from that for $\tau$ by moving beads up and down the runners so that the total number of moves to lower numbered positions is equal to the total number of moves to higher numbered positions, and the highest bead that is displaced is moved to a lower numbered position.

Let the $\beta$-numbers of $\tau$ on the 0 th runner be $0, p, \ldots,(r-1) p$, so that the highest $\beta$-number of $\tau$ is either $(r-1) p$ or else another number on the $r$ th row of the abacus. Then all the $\beta$-numbers of $\sigma$ must lie on the first $r$ rows.

In particular, no bead on the 0th runner can be moved. Thus the 0th component of the quotient of $\sigma$ is 0 , and since its abacus representation does not have $p$ consecutive gaps, $\sigma$ is column regular. Hence $\tau$ is strongly column regular.

Proposition 3.5. If the quotient of $\lambda$ has only one nonzero component $\alpha$, and if the corresponding component of the quotient of $\mu$ is nonzero, then $\left\langle s_{\lambda}^{\prime}, s_{\mu}\right\rangle=0$. Proof. First consider the special case where the core of $\lambda$ is

$$
((l-1)(p-1),(l-2)(p-1), \ldots, p-1),
$$

where $l=l(\alpha)$, and the quotient, with $b \equiv l \bmod p$, is $(\alpha,(0), \ldots,(0))$, as in Proposition 3.4. Let $s_{\lambda}^{\prime}=\sum_{\sigma} m_{\lambda, \sigma} s_{\sigma}$, where the sum is over all $\sigma$ in the same block such that $\sigma \leq \tau$, where $\tau$ is the highest weight of $s_{\lambda}^{\prime}$. By Proposition 3.4 , the 0th component of the quotient of each of these partitions $\sigma$ is 0 , so $m_{\lambda, \mu}=\left\langle s_{\lambda}^{\prime}, s_{\mu}\right\rangle=0$ for any other partition $\mu$.

However, by Corollary 2.10 , the statement that $\left\langle s_{\lambda}^{\prime}, s_{\mu}\right\rangle=0$ depends only on the quotients of $\lambda$ and $\mu$, and also remains true if the parts of the quotients of $\lambda$ and $\mu$ are permuted in the same way. Hence the special case implies the general case.

In general, it seems to be difficult to determine the highest weight of $s_{\lambda}^{\prime}$. We next consider a special case for which we are able to solve this problem, that in which $\lambda$ is horizontal.

Recall (Section 0) that we define the partition $\lambda$ to be horizontal if for each column of the diagram either all or none of the hook lengths is divisible by $p$. This condition has a simple interpretation in terms of cores and quotients.

Lemma 3.6. The partition $\lambda$ is horizontal if and only if $\lambda=\nu+p \alpha$, where the core of $\lambda$ is $\nu$, one component of its quotient is $\alpha$ and all others are (0), and $\nu_{i}-\nu_{i+1}=p-1$ for $1 \leq i<l(\alpha)$.

Proof. Assume $\lambda$ is horizontal. If no node has hook length divisible by $p$, then $\lambda$ is a core, so the statement holds with $\lambda=\nu$ and $\alpha=(0)$. Otherwise, let the first column whose hook lengths are divisible by $p$ be the $s$ th, and let the $(r, s)$ node be the lowest node in this column. Since all the hook lengths in this column are congruent $\bmod p$, the highest $r \beta$-numbers of $\lambda$ are also congruent $\bmod p$.

Now remove the first $s-1$ columns of the diagram of $\lambda$, to leave the diagram of a partition $\mu$ of length $r$ which is also horizontal. Since its first column hook lengths are all divisible by $p, \mu$ can be represented on an abacus by $r$ 
beads, all on the 0th runner. Hence the core of $\mu$ is the Steinberg partition $\bar{\nu}=((r-1)(p-1),(r-2)(p-1), \ldots, p-1)$ represented by moving these beads to positions $0, p, \ldots,(r-1) p$, and the quotient is $(\alpha,(0), \ldots,(0))$, where $\alpha$ has length $r$. Since there are no beads on any other runners, all the bead moves must correspond to removal of $p$-hooks of height 1 , i.e. hooks which consist of $p$ nodes in the same row. Hence $\mu=\bar{\nu}+p \alpha$.

Replacing the first $s-1$ columns, we can remove the same $p$-hooks from the diagram of $\lambda$, to leave a diagram $\nu$ such that $\lambda=\nu+p \alpha$. This process does not change the residue classes $\bmod p$ of any of the hook lengths, and so, since no hook length in the first $s-1$ columns of $\lambda$ is divisible by $p$, the same is true for $\nu$. Hence none of the hook lengths of the diagram of $\nu$ are divisible by $p$, and so $\nu$ is the core of $\lambda[8,2.7 .40]$. It follows that $\alpha$ is one component of the quotient of $\lambda$, and that every other component is $(0)$.

Conversely, it is easy to check that a partition of the form $\nu+p \alpha$ described in the lemma is horizontal.

Remark 3.7. It follows from the proof of Lemma 3.6 that a horizontal partition may equivalently be defined as one for which every sequence of removals of $p$-hooks from its diagram consists of successive removals of $p$ nodes from a single row. Lemma 3.6 also gives a convenient way to recognise horizontal partitions using the abacus representation. If the highest $r$ beads of the abacus representation of the core $\nu$ all lie on the same runner, then any partition represented by moving these beads is horizontal. Conversely, if a partition is horizontal, then the abacus moves required to represent the core are all on the same runner and no bead moves to a position lower than any bead on another runner.

Proposition 3.8. If $\lambda$ is horizontal and $\left\langle s_{\lambda}^{\prime}, s_{\mu}\right\rangle \neq 0$, then $\mu$ is strongly column regular.

Proof. We use Lemma 3.6. Consider the abacus representation of $\nu$, the core of $\lambda$, using $b$ beads, where $b \equiv l \bmod p$, and $l$ is the length of the nonzero component $\alpha$ of the quotient of $\lambda$. This is not necessarily the 0th component: in fact it is the $\nu_{l}$ th, but for simplicity we shall refer to the corresponding runner as the ' $\alpha$-runner'. Thus the highest $l$ beads (for $\nu$ ) lie on this runner, and by moving them down the abacus we obtain a representation of $\lambda$.

If $\left\langle s_{\lambda}^{\prime}, s_{\mu}\right\rangle \neq 0$, then by Proposition 3.5 the abacus representation of $\mu$ is obtained from that of $\nu$ by moving beads on the runners excluding the $\alpha$ runner. Thus all the movable beads lie below the $l$ th bead on the $\alpha$-runner. The moves are further restricted by Lemma 2.11 , which shows that no bead may be moved more than $l$ places. Hence in the abacus representation of $\mu$, they must lie below the first gap on the $\alpha$-runner. Thus there is no sequence of $p$ consecutive gaps below the top bead, i.e. $\mu$ is column regular.

Now let $\sigma$ be a partition in the same block as $\mu$, with $\sigma \leq \mu$. Since the highest bead in the abacus representation of $\sigma$ is not higher than that for $\mu$, it too lies below the first gap on the $\alpha$-runner in the representation of $\nu$. Hence no bead on the $\alpha$-runner can be moved in deriving the abacus representation of $\sigma$ from that of $\nu$, i.e. this component of the quotient of $\sigma$ is also (0). In the same way as before, it follows that $\sigma$ is also column regular. Hence $\mu$ is strongly column regular. 
Noting the result on the quotient of $\sigma$ proved in the course of this argument, we can restate Proposition 3.8 as a generalisation of Proposition 3.4 to arbitrary cores.

Corollary 3.9. For any horizontal partition $\lambda$, the highest weight $\rho=\rho(\lambda)$ of $s_{\lambda}^{\prime}$ is strongly column regular.

On an abacus where the kth component of the quotient of $\lambda$ is not $(0)$, the $k$ th component of the quotient of any partition $\sigma \leq \rho$ in the same block is (0) .

It remains to identify the highest weight of $s_{\lambda}^{\prime}$ when $\lambda$ is horizontal. The example of Remark 3.3 shows that the bound $\tau(\lambda)$, which solves this problem for row stable $\lambda$, is not achieved in general in the horizontal case.

Proposition 3.10. Let $\lambda$ be a partition with core $\nu$ whose quotient has only one nonzero component $\alpha$, and let $k=\alpha_{1}=l\left(\alpha^{\prime}\right)$. Let $c_{1} \geq c_{2} \geq \cdots \geq c_{k}$ be the $k$ highest $\beta$-numbers of $\nu$ which are not congruent $\bmod p$ to the highest one. Then the highest weight of $s_{\lambda}^{\prime}$ is the partition $\rho=\rho(\lambda)$ whose $\beta$-numbers are obtained from those of $\nu$ by replacing $c_{i}$ by $p \alpha_{i}^{\prime}+c_{i}$ for $1 \leq i \leq k$, i.e. the ith bead is moved $\alpha_{i}^{\prime}$ places on its runner.

If $\lambda$ is horizontal, then $\left\langle s_{\lambda}^{\prime}, s_{\rho}\right\rangle=1$, and in general $\left\langle s_{\lambda}^{\prime}, s_{\rho}\right\rangle=(-1)^{w} \varepsilon(\lambda) \varepsilon(\rho)$, where $w$ is the weight of $\lambda$.

This result may be summarised as follows: the parts of the components of the new quotient are the parts of the conjugate of the old quotient, and the core determines their distribution between the components. We illustrate the mechanism of this algorithm by an example.

Example 3.11. Let $p=3, \lambda=\left(13,5,1^{2}\right)$. Taking 7 beads on the abacus (any number $\geq 7$ will do), the $\beta$-numbers of $\lambda$ are $(19,10,5,4,2,1,0)$ and the 3 -quotient is $((0),(3,1),(0))$. The $\beta$-numbers of the core $\nu$ are $(10,7,5,4,2,1,0)$. Thus $\alpha^{\prime}=\left(2,1^{2}\right)$, and the core beads not on the 1runner are 5,2 , and 0 . We are supposed to move the bead in position 5 two places down and those in positions 2 and 0 one place down, to obtain the $\beta$-numbers $(11,10,7,5,4,3,1)$ for $\rho$. Hence $\rho=\left(5^{2}, 3,2^{3}, 1\right)$.

In this example, $\lambda$ is not row stable, and $\tau(\lambda)=\left(6,4,3,2^{3}, 1\right)$ is in the wrong block.

Proof of Proposition 3.10. The highest weight of $s_{\lambda}^{\prime}$ is the maximal partition $\mu$ in lexicographic order such that $\left\langle s_{\lambda}^{\prime}, s_{\mu}\right\rangle \neq 0$. By Proposition 3.5, we may assume that the component of the quotient of $\mu$ on the $\alpha$-runner is (0). Hence the sum in Proposition 2.7 has only one term, corresponding to the case where $\nu$ is the core of $\lambda$ and of $\mu$. Using Proposition 2.9, it follows that if $\left\langle s_{\lambda}^{\prime}, s_{\mu}\right\rangle \neq 0$ then

$$
\left\langle s_{\lambda}^{\prime}, s_{\mu}\right\rangle=(-1)^{w} \varepsilon(\lambda) \varepsilon(\mu)\left\langle s_{\alpha^{\prime}}, \prod_{k=0}^{p-1} s_{\mu(k)}\right\rangle,
$$

where $\mu(k)$ is the component of the quotient of $\mu$ on the $k$ th runner.

Applying the isometry $\omega$ to the scalar product, we have

$$
\left\langle s_{\alpha^{\prime}}, \prod_{k=0}^{p-1} s_{\mu(k)}\right\rangle=\left\langle s_{\alpha}, \prod_{k=0}^{p-1} s_{\mu(k)^{\prime}}\right\rangle .
$$


Since $s_{\mu(k)^{\prime}}$ has highest weight $\mu(k)^{\prime}, \prod_{k=0}^{p-1} s_{\mu(k)^{\prime}}$ has highest weight $\beta^{\prime}=$ $\sum_{k=0}^{p-1} \mu(k)^{\prime}$, where [13, p. 5] $\beta$ is the partition whose parts are those of the partitions $\mu(k), 0 \leq k \leq p-1$, arranged in descending order. Further, since other partitions $\sigma$ with nonzero coefficient $K_{\mu(k)^{\prime}, \sigma}$ in the expansion $s_{\mu(k)^{\prime}}=$ $\sum K_{\mu(k)^{\prime}, \sigma} m_{\sigma}$ are dominated by $\mu(k)^{\prime}$, all partitions with nonzero coefficient in $\prod_{k=0}^{p-1} s_{\mu(k)^{\prime}}$ are dominated by $\beta^{\prime}$. Hence $\alpha \unlhd \beta^{\prime}$. This is equivalent to $\alpha^{\prime} \unrhd \beta[13, \mathrm{I}(1.11)]$, and hence $\alpha^{\prime} \geq \beta$. Thus we have proved

$$
\left\langle s_{\alpha^{\prime}}, \prod_{k=0}^{p-1} s_{\mu(k)}\right\rangle= \begin{cases}0, & \text { if } \beta>\alpha^{\prime}, \\ 1, & \text { if } \beta=\alpha^{\prime} .\end{cases}
$$

Now let $\rho$ denote the particular choice of $\mu$ obtained by moving the highest $k$ beads in the abacus representation of the core $\nu$ (excluding those on the $\alpha$ runner) in succession by $\alpha_{1}^{\prime}, \alpha_{2}^{\prime}, \ldots, \alpha_{k}^{\prime}$ places respectively on their runners. By considering the abacus representation, it is easy to see that $\rho$ is the maximal partition in lexicographic order such that $\beta=\alpha^{\prime}$, and that $\rho \geq \mu$ for any partition $\mu$ for which the partition $\beta$ constructed as above from the quotient of $\mu$ satisfies $\beta<\alpha^{\prime}$. Thus $\rho$ is the maximal partition in lexicographic order such that $\left\langle s_{\lambda}^{\prime}, s_{\rho}\right\rangle \neq 0$, and in fact $\left\langle s_{\lambda}^{\prime}, s_{\rho}\right\rangle=(-1)^{w} \varepsilon(\lambda) \varepsilon(\rho)$.

Finally, when $\lambda$ is horizontal, the sign $\varepsilon(\lambda)=1$ and $\varepsilon(\rho)=(-1)^{w}$. The first statement follows from Lemma 3.6, since the abacus representation for $\lambda$ is obtained from that for $\nu$ by moving the top $r$ beads on the $\alpha$-runner, so that no beads on other runners are passed in this process. The second statement follows from the fact that, in moving the top $k$ beads on other runners in the abacus representation for $\nu$, a bead on the $\alpha$-runner, and no other bead, is passed at each of the $w$ steps. Hence $\left\langle s_{\lambda}^{\prime}, s_{\rho}\right\rangle=1$ when $\lambda$ is horizontal.

We conclude this section by showing that when $\lambda$ is row stable the partition $\tau=\tau(\lambda)$ of Proposition 3.1 is the transpose of the row regular partition associated to $\lambda$ by the algorithm of Mullineux. We write $M$ for Mullineux's involution [15] on the set of all row regular partitions. We omit the definition of $M$, but provide an example for the reader's assistance.

Example 3.12. Let $p=3$ and $\lambda=(8,5,3)$, so that $\tau=(6,5,3,2)$. The Mullineux numbering of the diagrams of $\lambda$ and $\tau$ is shown below.

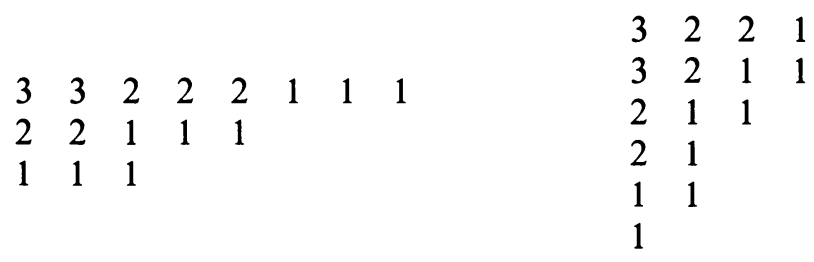

Proposition 3.13. If $\lambda$ is row stable, then $M(\lambda)=\tau^{\prime}$, where $\tau$ is as in Proposition 3.2 .

Proof. Consider the first Mullineux rim of the diagram of $\lambda$, i.e. the nodes numbered by 1 's. The stability condition shows that this rim consists of the last $p$ nodes in each row (except possibly the last row, which will lie entirely in this rim if $\left.\lambda_{l}<p\right)$. Removal of this rim clearly leaves a row stable subdiagram, so by repeating the argument we see that all the Mullineux rims have this form. Thus the Mullineux numbering is obtained by numbering off each row of the 
diagram of $\lambda$ from the end by $p$ 1's, $p 2$ 's, and so on, the number of nodes in the last-numbered group at the beginning of the $i$ th row being only the residue of $\lambda_{i} \bmod p$.

This numbering is the same as we would obtain by regarding each row of the diagram of $\lambda$ as a separate diagram $\lambda_{i}$, numbered by Mullineux's procedure. Now it is easy to check that $M\left(\lambda_{i}\right)=\tau\left(\lambda_{i}\right)^{\prime}$. Thus we may number the diagram of $\tau^{\prime}$ in such a way that the $i$ th block of $p-1$ rows is numbered by the same number of 1 's, 2 's etc. as were used for numbering $\lambda_{i}$. It is also easy to see that the successive Mullineux rims of $\tau\left(\lambda_{i}\right)^{\prime}$ for $1 \leq i \leq l$ fit together to form the successive Mullineux rims of $\tau^{\prime}$ (again, one need only check the first rim). Moreover, each group of $p$ like-numbered nodes within a block forms a $p$-hook which has at least one node in each row of the block, and so has two nodes in exactly one row. These conditions imply that this numbering of the diagram of $\tau^{\prime}$ is the one given by Mullineux's algorithm.

\section{SOME SIMPLE CHARACTERS OF $G L(n)$}

We now restrict the modulus $p$ to be prime. Our object is to relate the modular Schur functions $s_{\lambda}^{\prime}$ to the modular representation theory of the general linear group $G L(n, K)$ in the natural characteristic $p$ of the field $K$. We shall consider (polynomial) left modules over the group algebra $K G L(n, K)$, where $K$ is an infinite field of characteristic $p>0$.

The main result of this section is Theorem 4.4, which gives a sufficient condition for $s_{\lambda}^{\prime}$ to be a simple character of $G L(n, K)$. This condition is the Carter criterion, which was proved by James to be a necessary and sufficient condition for the Specht module $S^{\lambda}$ for a row regular partition $\lambda$ to give an irreducible modular representation of $S_{d}$, where $d=|\lambda|$. Theorem 4.4 is in effect a corollary of this theorem of James.

For each prime $p$, the ring of symmetric functions $\Lambda_{n}$ has a very important Z-basis, namely the set of simple (formal) polynomial characters $l_{\lambda}$ of $G L(n, K)$ (cf. [14, Theorem 1.6.1; 4, p. 14]), indexed by the partitions $\lambda$ of length $\leq n$, where $l_{\lambda}$ has highest weight $\lambda$. The simple characters $l_{\lambda}$ are not known in general, but it is known that they behave in the same way as the Schur functions with respect to the projection $\Lambda_{n} \rightarrow \Lambda_{n-1}$; i.e. $l_{\lambda}$ maps to $l_{\lambda}$ if $l(\lambda)<n$ and to 0 if $l(\lambda)=n$. (This can be seen using either of the standard descriptions of the simple $G L(n, K)$ module $L(\lambda)$, namely as the quotient of the Weyl module $V(\lambda)$ by the submodule $V(\lambda) \cap V(\lambda)^{\perp}[8,8.3 .3]$, or as the submodule of the Schur module $H^{0}(\lambda)$ generated by a suitable 'bideterminant' $\left[5\right.$, p. $72 ; 14$, Theorem 3.4.1].) Hence the functions $l_{\lambda}$ define a Z-basis of $\Lambda$, indexed by the set of all partitions. The coefficients in the expansion of a symmetric function $f$ with respect to this basis are called the decomposition numbers of $f$. If $f$ is homogeneous of degree $d$, then all the decomposition numbers of $f$ are obtained by working in $\Lambda_{n}$ for any $n \geq d$, since the decomposition numbers of $f$ for $n<d$ are the subset of these which are indexed by partitions of length $\leq n$.

These decomposition numbers have been studied mainly for the case where $f$ is a Schur function, when they describe the composition factors of the (Schur or Weyl) modules for $G L(n, K)$. Thus, for each $d$, we have a 'decomposition matrix' $D=d_{\lambda, \mu}(G L(n))$ which records the numbers $d_{\lambda, \mu}(G L(n))=$ 
$[V(\lambda): L(\mu)]$ for all partitions $\lambda$ and $\mu$ of degree $d$. An important theorem of James [7] states that the 'decomposition matrix' of the symmetric group $S_{d}$, which expresses the modular reduction of the irreducible ordinary characters of $S_{d}$ in terms of the irreducible modular characters, is the submatrix of $D$ defined by the column regular partitions $\mu$. More precisely [14, Theorem 4.4.3], when $\mu$ is column regular,

$$
d_{\lambda, \mu}(G L(n))=\left[S_{\lambda}: D_{\mu}\right],
$$

where $S_{\lambda}$ is the dual Specht module over $K$, and $D_{\mu}$ is the irreducible quotient of $S_{\mu}$.

With the understanding that $n \geq d$, we write $d_{\lambda, \mu}=d_{\lambda, \mu}(G L(n))$, so that $s_{\lambda}=\sum_{\mu} d_{\lambda, \mu} l_{\mu}$. Since we wish to study the decomposition numbers of the modular Schur functions $s_{\lambda}^{\prime}$, we similarly write $s_{\lambda}^{\prime}=\sum_{\mu} d_{\lambda, \mu}^{\prime} l_{\mu}$. In general, the function $s_{\lambda}^{\prime}$ is only a virtual character of $G L(n, K)$, i.e. its decomposition numbers $d_{\lambda, \mu}^{\prime}$ can be negative, whereas of course $d_{\lambda, \mu} \geq 0$.

Example 4.1. In the case of a block of weight 1 the decomposition matrix $M(s, l)$ is well known from Brauer's classical work (see e.g. $[14, \S 5.6]$. The block of Example 1.11 is typical: with the $p=5$ partitions in the block in lexicographic order, the matrix is

$$
M(s, l)=\left(\begin{array}{lllll}
1 & 0 & 0 & 0 & 0 \\
1 & 1 & 0 & 0 & 0 \\
0 & 1 & 1 & 0 & 0 \\
0 & 0 & 1 & 1 & 0 \\
0 & 0 & 0 & 1 & 1
\end{array}\right)
$$

Hence the decomposition matrix for modular Schur functions in this block is

$$
M\left(s^{\prime}, l\right)=M\left(s^{\prime}, s\right) M(s, l)=\left(\begin{array}{rrrrr}
1 & 0 & 0 & 0 & -1 \\
1 & 1 & 0 & 0 & 1 \\
0 & 1 & 1 & 0 & -1 \\
0 & 0 & 1 & 1 & 1 \\
0 & 0 & 0 & 1 & 0
\end{array}\right),
$$

where the last column corresponds to the unique column singular partition in the block, and is of course identical with the last column of $M\left(s^{\prime}, s\right)$. The other columns, which correspond to column regular partitions, are the same as the corresonding columns of $M(s, l)$.

We next show that this coincidence of $d_{\lambda, \mu}^{\prime}$ with $d_{\lambda, \mu}$ when $\mu$ is column regular is true generally.

Proposition 4.2. For any prime $p$ and any partition $\lambda, s_{\lambda}^{\prime}$ and $s_{\lambda}$ have the same column regular decomposition numbers.

Proof. Recall [13, I.7] the characteristic map ch, which gives a bijection between Z-linear combinations of the ordinary irreducible characters $\chi^{\lambda}$ of $S_{d}$ and homogeneous symmetric functions of degree $d$ in $\Lambda$, such that $\operatorname{ch}\left(\chi^{\lambda}\right)=s_{\lambda}=$ $\sum_{\rho} z_{\rho}^{-1} \chi_{\rho}^{\lambda} P_{\rho}$.

By James's theorem, we may obtain the column regular decomposition numbers for any homogeneous symmetric function $f$ of degree $d$ by writing $\mathrm{ch}^{-1}(f)$ as a $\mathbf{Z}$-linear combination of the irreducible characters $\chi^{\mu}$, i.e. as 
a virtual (ordinary) character of $S_{d}$, and applying modular reduction. From $[13, \mathrm{I}(7.2)]$ the value of this virtual character on the conjugacy class $C(\rho)$ of elements of cycle type $\rho$ in $S_{d}$ is given by expressing $f$ in terms of the power sum basis for $\Lambda \otimes_{Z} \mathbf{Q}$, i.e.

$$
f=\sum_{|\rho|=d} z_{\rho}^{-1} \mathrm{ch}^{-1}(f)_{\rho} P_{\rho} .
$$

But, applying $\omega^{\prime}$ to the formula for $s_{\lambda}$ above, we have $s_{\lambda}^{\prime}=\sum_{\rho} z_{\rho}^{-1} \chi_{\rho}^{\lambda} P_{\rho}^{\prime}$. Using Proposition 1.2, $P_{\rho}^{\prime}=P_{\rho}$ if $C(\rho)$ is a $p$-regular conjugacy class, and $P_{\rho}^{\prime}=(1-p)^{w(\rho)} P_{\rho}$, where $w(\rho)$ is a positive integer, if $C(\rho)$ is $p$-singular.

Thus the virtual character $\mathrm{ch}^{-1} s_{\lambda}^{\prime}$ takes the same value on every $p$-regular class $C(\rho)$ as the irreducible character $\operatorname{ch}^{-1} s_{\lambda}=\chi^{\lambda}$. Now, by the theory of Brauer characters, two ordinary representations of $S_{d}$ have the same modular reduction if and only if they take the same values on all $p$-regular conjugacy classes of $S_{d}$.

Corollary 4.3. If $\lambda$ is horizontal, then

$$
d_{\lambda, \mu}^{\prime}= \begin{cases}d_{\lambda, \mu}, & \text { if } \mu \text { is column regular } \\ 0, & \text { if } \mu \text { is column singular } .\end{cases}
$$

Proof. This follows immediately by combining Proposition 4.2 with Corollary 3.9.

Before stating the main theorem, we must explain the indexing that occurs. If $s_{\lambda}^{\prime}$ is simple, then we wish to know its highest weight $\mu$, so as to identify $s_{\lambda}^{\prime}$ with the simple character $l_{\mu}$.

Recall that there are two standard ways to index the irreducible $p$-modular representations of the symmetric group. They are the simple quotients $D^{\lambda}$ of the Specht modules $S^{\lambda}$, for row regular $\lambda$. They are also the simple quotients $D_{\mu}$ of the dual Specht modules $S_{\mu}$, for column regular $\mu$. The two indexings are related by the fact that $S_{\lambda} \cong S^{\lambda^{\prime}} \otimes K_{a}$, where $K_{a}$ is the 1-dimensional module giving the sign character of $S_{d}$. (See [8, p. 318] or [14, §4.2].) Now we have an involution $l$ on the set of all row regular partitions of degree $d=|\lambda|$ defined by $D^{\prime(\lambda)} \cong D^{\lambda} \otimes K_{a}$. It follows that $D^{\lambda} \cong D_{\mu}$, where $\mu=l(\lambda)^{\prime}$. Moreover, the Schur module $H^{0}(\lambda)$ has a unique simple quotient $L(\mu)$ [2, (2.10)], [10]. Thus it is reasonable to regard $l_{\mu}$ as a function of $\lambda$ : our theorem states that when $\lambda$ is horizontal, this function is $s_{\lambda}$.

Theorem 4.4. Let $K$ be an arbitrary field of characteristic $p$, and let $\lambda$ satisfy the Carter condition. Then the modular Schur function $s_{\lambda}^{\prime}$ is the simple character $l_{\mu}$ of $G L(n, K)$ over $K$ for any $n \geq l$, where $\mu=l(\lambda)^{\prime}$ has length $l$. Conversely, if $\lambda$ is row regular but does not satisfy the Carter condition, then $s_{\lambda}^{\prime}$ is not a simple character of $G L(n, K)$ for all sufficiently large $n$.

Proof. We first note that it is sufficient to prove both statements for the case where $K$ is infinite. Since $\lambda$ is row regular, $\mu$ is column regular, and hence the simple character $l_{\mu}$ for $K$ infinite remains a simple character on restriction to $F_{p}$.

We prove the positive statement first. For this, it suffices to work with $n \geq$ $|\lambda|$, since the result follows for smaller values of $n$ from the fact that both $s_{\lambda}^{\prime}$ and $l_{\mu}$ project to the corresponding functions in a smaller number of variables. 
A partition satisfying the Carter condition is horizontal, so, by Corollary 4.3, we are reduced to proving that $d_{\lambda, \mu}=1$ if $\mu=\imath(\lambda)^{\prime}$, and $d_{\lambda, \mu}=0$ for all other column regular partitions $\mu$. By James's theorem on decomposition numbers, this is equivalent to the statement that the dual Specht module $S_{\lambda}=D_{\mu}$. By the remarks above on the indexing of simple $K S_{d}$-modules, this is equivalent to $S_{\lambda}=D^{\lambda}$. Again, since $S^{\lambda}$ and $S_{\lambda}$ are dual modules in the usual sense [8, p. $318 ; 5$, p. 92], we are reduced to proving that the Specht module $S^{\lambda}$ is simple. A famous theorem of James and Murphy [6,9] (see also [8, 7.3.23; 14, Theorem 4.4.5]) assures us that this is so: $\lambda$ is row regular and $S^{\lambda}$ is simple if, and only if, $\lambda$ satisfies the Carter condition.

The converse statement follows from the 'only if' part of James's theorem. If $\lambda$ is row regular but does not satisfy the Carter condition, then $S^{\lambda}$ has at least two composition factors, and, in the same way as before, it follows that $d_{\lambda, \mu}^{\prime} \neq 0$ for at least two column regular partitions $\mu$.

Remark 4.5. In the case $p=2$, Theorem 4.4 gives no new simple characters of $G L(n, K)$. In this case, $\mu=\lambda^{\prime}$ and $s_{\lambda}^{\prime}=s_{\mu}$. Likewise, when $\lambda$ is a core, $s_{\lambda}^{\prime}=s_{\lambda}$ by Proposition 1.8, so we do not get a new simple character: in this case $\mu=\lambda$ by a theorem of Mullineux [16].

A special case of Theorem 4.4 for certain horizontal partitions of weight 1 was conjectured in [3, Remark 2.13].

Conjecture 4.6. If $\lambda$ does not satisfy the Carter condition, then $s_{\lambda}^{\prime}$ is not a simple character of $G L(n, K)$ over $K$ when $n$ is sufficiently large.

If this conjecture is true, then $s_{\lambda}^{\prime}$ is a simple $G L(n, K)$ module for $n \geq|\lambda|$ if and only if the same is true for $s_{\lambda^{\prime}}$. One way to prove the conjecture would be to show that the highest weight of $s_{\lambda}^{\prime}$ is column singular when $\lambda$ is row singular. Although computations suggest that this is plausible, it does not seem easy to give a lower bound on the highest weight of $s_{\lambda}^{\prime}$ analogous to the upper bound $\tau(\lambda)$ of Lemma 3.1. Hence a proof of Conjecture 4.6 remains elusive.

Consider, for example, the case where $\lambda$ is column regular. Since $S_{\lambda} \cong$ $S^{\lambda^{\prime}} \otimes K_{a}, S_{\lambda}$ is simple if and only if $S^{\lambda^{\prime}}$ is simple. Hence the argument used in the proof of Theorem 4.4 rules out the possibility that $s_{\lambda}^{\prime}$ is simple, except in the case where $\lambda^{\prime}$ satisfies the Carter condition. This would of course imply that $s_{\lambda^{\prime}}=l_{\lambda^{\prime}}$, by James's theorem. Hence, if $s_{\lambda}^{\prime}$ is simple, we would require $s_{\lambda}^{\prime}=s_{\lambda}$. In Proposition 1.8, we have already observed that this holds when $\lambda$ is a core: of course, in this case $\lambda$ as well as $\lambda^{\prime}$ satisfies the Carter condition, so Theorem 4.4 applies. This suggests a further conjecture.

Conjecture 4.7. For all $p \geq 2$ and $n \geq 1$, if $s_{\lambda}^{\prime}=s_{\lambda}$ then $\lambda$ is a core.

This would follow from a similar argument to that of Proposition 4.2, if it can be shown that $\lambda$ must be a core when the irreducible ordinary character $\chi^{\lambda}$ of $S_{d}$ vanishes on all $p$-singular conjugacy classes.

If we take $n<l(\mu)$ in Theorem 4.4, then in $\Lambda_{n}, l_{\mu}=0$, and hence $s_{\lambda}^{\prime}=0$. Usually this happens because $\lambda_{1}>n(p-1)$, but not always: for example, let $n=p=3$, and let $\lambda$ be the core $\left(4,2,1^{2}\right)$. It is clearly useful to have a means of evaluating $l(\mu)$.

When $\lambda$ is horizontal, $\mu=\imath(\lambda)^{\prime}$ can be identified with the highest weight $\rho$ of $s_{\lambda}^{\prime}$ given by Proposition 3.10. This algorithm shows that, for a given core 
and sufficiently large quotients, addition of $p(p-1)$ nodes to the first part of $\lambda$ corresponds to the addition of $p$ parts $p-1, p-1, \ldots, p-1$ to $\rho$. Thus the growth rate of $\lambda_{1}$ is $p-1$ times the growth rate of $l(\mu)$. This is further evidence that the set of partitions considered in Conjecture 1.4 is a natural object of study.

\section{REFERENCES}

1. D. P. Carlisle and N. J. Kuhn, Subalgebras of the Steenrod algebra and the action of matrices on truncated polynomial algebras, J. Algebra 121 (1989), 370-387.

2. S. Donkin, On Schur algebras and related algebras. II, J. Algebra 111 (1987), 354-364.

3. S. R. Doty and G. Walker, The composition factors of $F_{p}\left[x_{1}, x_{2}, x_{3}\right]$ as a $G L(3, p)$ module, J. Algebra 147 (1992), 411-441.

4. ear groups, J. Pure Appl. Algebra 82 (1992), 1-26.

5. J. A. Green, Polynomial representations of $G L(n)$, Lecture Notes in Math., vol. 830, Springer, 1980.

6. G. D. James, On a conjecture of Carter concerning irreducible Specht modules, Math. Proc. Cambridge Philos. Soc. 83 (1978), 11-17.

7. $ـ$ The decomposition of tensors over fields of prime characteristic, Math. Z. 172 (1980), 161-178.

8. G. D. James and A. Kerber, The representation theory of the symmetric group, Encyclopaedia of Mathematics and its Applications 16, Addison-Wesley, Reading, MA, 1981.

9. G. D. James and G. E. Murphy, The determinant of the Gram matrix for a Specht module, J. Algebra 59 (1979), 222-235.

10. F. W. Kouwenhoven, Schur and Weyl functors. II, Comm. Algebra 18 (1990), 2885-2941.

11. D. E. Littlewood, The theory of group characters, 2nd ed., Clarendon Press, Oxford, 1950.

12. _ Modular representations of symmetric groups, Proc. Roy. Soc. London Ser. A 209 (1951), 333-353.

13. I. G. Macdonald, Symmetric functions and Hall polynomials, Oxford Univ. Press, 1979.

14. S. Martin, Schur algebras and representation theory, Cambridge Univ. Press, 1993.

15. G. Mullineux, Bijections of p-regular partitions and p-modular irreducibles of the symmetric groups, J. London Math. Soc. 20 (1979), 60-66.

16. _ On the p-cores of regular diagrams, J. London Math. Soc. 20 (1979), 222-226.

17. J. C. Scopes, Cartan matrices and Morita equivalence for blocks of the symmetric groups, J. Algebra 142 (1991), 441-455.

Department of Mathematics, University of Manchester, Oxford Road, Manchester M13 9PL, UNITED KINGDOM

E-mail address: grant@ma.man.ac.uk 